\title{
The Mitochondria-Derived Peptide Humanin Improves Recovery from Intracerebral Hemorrhage: Implication of Mitochondria Transfer and Microglia Phenotype Change
}

\author{
Joo Eun Jung, @Guanghua Sun, Jesus Bautista Garrido, Lidiya Obertas, Alexis S. Mobley, Shun-Ming Ting, \\ Xiurong Zhao, and $\odot$ Jaroslaw Aronowski \\ Department of Neurology, University of Texas Health Science Center at Houston, McGovern Medical School, Houston, Texas 77030
}

\begin{abstract}
Astrocytes are an integral component of the neurovascular unit where they act as homeostatic regulators, especially after brain injuries, such as stroke. One process by which astrocytes modulate homeostasis is the release of functional mitochondria (Mt) that are taken up by other cells to improve their function. However, the mechanisms underlying the beneficial effect of Mt transfer are unclear and likely multifactorial. Using a cell culture system, we established that astrocytes release both intact Mt and humanin (HN), a small bioactive peptide normally transcribed from the Mt genome. Further experiments revealed that astrocyte-secreted Mt enter microglia, where they induce $\mathrm{HN}$ expression. Similar to the effect of $\mathrm{HN}$ alone, incorporation of Mt by microglia (1) upregulated expression of the transcription factor peroxisome proliferator-activated receptor gamma and its target genes (including mitochondrial superoxide dismutase), (2) enhanced phagocytic activity toward red blood cells (an in vitro model of hematoma clearance after intracerebral hemorrhage [ICH]), and (3) reduced proinflammatory responses. ICH induction in male mice caused profound HN loss in the affected hemisphere. Intravenously administered HN penetrated perihematoma brain tissue, reduced neurological deficits, and improved hematoma clearance, a function that normally requires microglia/macrophages. This study suggests that astrocytic Mt-derived HN could act as a beneficial secretory factor, including when transported within Mt to microglia, where it promotes a phagocytic/reparative phenotype. These findings also indicate that restoring HN levels in the injured brain could represent a translational target for ICH. These favorable biological responses to $\mathrm{HN}$ warrant studies on $\mathrm{HN}$ as therapeutic target for ICH.
\end{abstract}

Key words: mitochondria; humanin; intracerebral hemorrhage; microglia; astrocyte; peroxisome proliferator-activated receptor gamma

Significance Statement

Astrocytes are critical for maintaining brain homeostasis. Here, we demonstrate that astrocytes secrete mitochondria (Mt) and the Mt-genome-encoded, small bioactive peptide humanin (HN). Mt incorporate into microglia, and both Mt and HN promote a "reparative" microglia phenotype characterized by enhanced phagocytosis and reduced proinflammatory responses. Treatment with HN improved outcomes in an animal model of intracerebral hemorrhage, suggesting that this process could have biological relevance to stroke pathogenesis.

\section{Introduction}

Intracerebral hemorrhage $(\mathrm{ICH})$ is a subtype of stroke caused by leaking or rupture of cerebral blood vessels within the brain parenchyma; it carries very high mortality rate $(30 \%-67 \%)$ and

\footnotetext{
Received Sept. 5, 2019; revised Jan. 7, 2020; accepted Jan. 11, 2020.

Author contributions: J.E.J. and J.A. designed research; J.E.J., G.S., J.B.G., L.O., A.S.M., and S.-M.T. performed research;J.E.J., L.O., A.S.M., S.-M.T., X.Z., and J.A. analyzed data; J.E.J. wrote the first draft of the paper; J.E.J., A.S.M., and J.A. edited the paper; J.E.J. wrote the paper.

This work was supported by National Institutes of Health Grant R01 NS111590-01.

The authors declare no competing financial interests.

Correspondence should be addressed to Joo Eun Jung at Joo.Eun.Jung@uth.tmc.edu or Jaroslaw Aronowski at J.Aronowski@uth.tmc.edu.

https://doi.org/10.1523/JNEUROSCI.2212-19.2020

Copyright $\odot 2020$ the authors
}

poor prognosis for recovery, with limited therapeutic options (Qureshi et al., 2009; Adeoye and Broderick, 2010). After ICH, extravasated blood has direct contact with the brain parenchyma where it acts as a "reservoir" of toxicity for brain tissue, leading to progressive secondary "(bio)chemical” injury (Felberg et al., 2002; Wagner et al., 2003; Xi et al., 2004; X. Zhao et al., 2009a) through various mechanisms, including oxidative insult and complex proinflammatory responses (Hickenbottom et al., 1999; Wu et al., 2002; Aronowski and Hall, 2005; Keep et al., 2012). Ultimately, the hematoma is cleared by microglia/macrophages $(\mathrm{M} \Phi)$ through phagocytosis, a process controlled by many factors, including peroxisome proliferator-activated receptor gamma (PPAR $\gamma$ ) (Ricote et al., 1999; X. Zhao et al., 2007b, 
2015c). This transcription factor induces the expression of key proteins controlling phagocytosis and associated cleanup processes after ICH, including antioxidative enzymes, such as superoxide dismutase (SOD) and catalase (X. Zhao et al., 2006, 2007b; Tureyen et al., 2007; Roszer et al., 2011). PPAR $\gamma$ also inhibits proinflammatory responses at multiple levels (Ricote et al., 1999; Sundararajan and Landreth, 2004; X. Zhao et al., 2007b).

Blood cell components (e.g., hemoglobin, heme, and iron) in hematomas are a major source of noxious free radicals that can cause oxidative damage to neighboring brain tissue (Regan and Panter, 1996; Felberg et al., 2002; Wagner et al., 2003; Nakamura et al., 2004; Aronowski and Hall, 2005). Impaired expression and dysfunction of antioxidative enzymes may amplify oxidative damage; for example, levels of the essential intramitochondrial antioxidant enzyme manganese SOD (Mn-SOD) are reduced in ICH-affected brain tissue (Wu et al., 2002). Since Mn-SOD shields mitochondria (Mt) and cells from oxidative damage, therapeutic approaches designed to upregulate Mn-SOD in the perihematoma zone could mitigate ICH-mediated damage.

Hayakawa et al. (2016) demonstrated that astrocytes transfer functional Mt to adjacent neurons, where they support prosurvival functions. However, the mechanism of how transferred Mt exert protective effects remains unclear. Mt are involved in myriad essential functions that can benefit recipient cells. Here we propose that one of the important tasks of transferred astrocytic $\mathrm{Mt}$ is supplying recipient cells with humanin $(\mathrm{HN})$, a small peptide transcribed from MtDNA. HN is a 24 -amino acid Mtderived peptide discovered in the brain of an Alzheimer's patient (Hashimoto et al., 2001; Guo et al., 2003). The rat HN homolog rattin $(\mathrm{HNr})$ is a 38 amino acid, and both $\mathrm{HN}$ and $\mathrm{HNr}$ are transcribed from an open reading frame within the $16 \mathrm{~S}$ ribosomal RNA locus of MtDNA and are translated in the Mt and cytoplasm (Matsuoka and Hashimoto, 2010; Paharkova et al., 2015). HN can be stored in Mt or secreted into the extramitochondrial and extracellular spaces as an Mt autocrine, paracrine, and endocrine signal (Matsuoka and Hashimoto, 2010; Yen et al., 2013). Extracellularly secreted HN activates a cytoprotective signal by binding to a cytokine-like trimeric receptor composed of CNFR/WSX1/gp130 (Hashimoto et al., 2009a,b).

HN's biological role has largely been studied in age-related contexts, including regulation of healthy aging, longevity, metabolism, and inflammation (Matsuoka, 2011; S. T. Zhao et al., 2013; Gong et al., 2014; Fuku et al., 2015; Kim et al., 2017). Less is known about $\mathrm{HN}$ in cerebrovascular diseases. It was shown to reduce brain infarction in a model of focal ischemia (Xu et al., 2006) and ameliorate neurological deficits in mice subjected to ICH (Wang et al., 2013). Here we demonstrate that astrocytes transfer Mt to microglia where, similar to $\mathrm{HN}$ alone, they promote "reparative" microglia function. This suggests that the beneficial effects of Mt transfer between astrocytes and microglia include generation of $\mathrm{HN}$, and this process could be relevant to the pathogenesis of ICH.

\section{Materials and Methods}

Animals. All animal studies were performed in accordance with the Guide for the care and use of laboratory animals from the National Institutes of Health guidelines and were approved by the Animal Welfare Committee of the University of Texas Health Science Center at Houston. All animal experiments and analyses used the approach of randomization, and investigators were blinded from treatments. Rodents were housed in standard cages under a $12 \mathrm{~h}$ inverted light-dark cycle. Three-month-old male C57BL/6J mice were purchased from The Jackson Laboratory, and female Sprague Dawley rats (embryonic $17 \mathrm{~d}$ timed pregnant) for primary cerebral cortical astrocyte and microglia cultures were purchased from Charles River Laboratories.

ICH. Intrastriatal injection of autologous blood was used to simulate human ICH in mice as we described previously (X. Zhao et al., 2007b, 2017). Briefly, male C57BL/6J mice (25-30 g) were anesthetized with chloral hydrate $(0.35 \mathrm{~g} / \mathrm{kg}$; i.p. $)$ and placed in a stereotaxic apparatus before a 1-mm-diameter burr hole was drilled in the skull at the following coordinates: $0.0 \mathrm{~mm}$ anterior to bregma, $3.0 \mathrm{~mm}$ lateral to midline. A 30 -gauge cannula was then inserted into the left striatum, $3.5 \mathrm{~mm}$ deep from the skull for blood (collected from femoral vein) infusion $(18 \mu \mathrm{l}$ at $1 \mu \mathrm{l} / \mathrm{min}$ ). The body temperature was maintained at $37 \pm 0.5^{\circ} \mathrm{C}$ during the procedure and for $2 \mathrm{~h}$ after surgery. Sham-operated mice underwent the same surgical procedures without blood infusion.

$H N$ administration. HN peptide was purchased from ANA SPEC (AS60886). C57BL/6J male mice were randomly assigned to ICH surgery and treatments. Mice received $\mathrm{HN}$ (one injection per mouse per day for a total of 7 administrations) either intraperitoneally ( $1 \mu \mathrm{g} / \mathrm{g}$ in PBS) or intranasally ( $1 \mu \mathrm{g} / \mathrm{g}$ in PBS), starting $60 \mathrm{~min}$ (i.p.) or $3 \mathrm{~h}$ (intranasal) after the onset of ICH. PBS was used as vehicle control. To assess whether systemic administered HN enters ICH-injured brain, FITC-conjugated $\mathrm{HN}(1 \mu \mathrm{g} / \mathrm{g}$ in PBS$)$ was injected into mice via femoral vein, $24 \mathrm{~h}$ after the onset of ICH. The HN-FITC peptide was customized/synthesized by WatsonBio Sciences.

Neurological deficit score (NDS) measurement. All mouse behavioral tests were conducted under quiet, low-light conditions. The behavioral outcomes were measured by an investigator blinded to the treatment groups before and on day 3 for intranasal intervention and on days 3, 7, and 14 for intraperitoneal intervention after ICH. Foot-fault, postural flexing, wire, and corner tests were used to calculate the NDS as we reported previously (X. Zhao et al., 2007b, 2009c, 2017). Pretesting excluded animals with a preference during corner testing and/or $>20 \%$ foot faults before being subjected to $\mathrm{ICH}$.

Brain tissue harvesting. Mice anesthetized with chloral hydrate $(0.5$ $\mathrm{g} / \mathrm{kg}$, i.p.) were transcardially perfused with ice-cold PBS. The brains were immediately frozen by submersion in 2 -methylbutane $\left(-80^{\circ} \mathrm{C}\right)$ and stored at $-80^{\circ} \mathrm{C}$ until cryo-sectioning or RNA or protein extraction.

Hematoma cleanup model (hematoma resolution). To assess hematoma resolution, the remaining amount of hemoglobin present in hematomaaffected brain tissue was measured on day 7 after ICH, as we described previously (X. Zhao et al., 2007b, 2009c, 2017). Briefly, anesthetized mice were transcardially perfused with ice-cold PBS to remove intravascular blood. Brains were collected, and coronal hematoma-containing sections were prepared using a brain matrix and razor blade. Following homogenization and centrifugation, hemoglobin in the supernatant was calorimetrically measured with Drabkin's reagent (D5941-6VL; Sigma Millipore) (X. Zhao et al., 2007b, 2009c, 2017). For a calibration curve, various volumes of peripheral blood were added to naive brain homogenates. The data are expressed as blood volume per brain homogenate.

Immunohistochemistry. Rodents were placed under chloral hydrate anesthesia and subjected to transcardial perfusion with ice-cold PBS followed by $4 \%$ PFA. Brain tissue was collected in 30\% sucrose solution for $24 \mathrm{~h}$ before snap freezing and cryosectioning at $20 \mu \mathrm{m}$. Sections were incubated in $0.1 \%$ Triton X-100 for $15 \mathrm{~min}$ at room temperature and blocked with PBS containing 3\% BSA for $1 \mathrm{~h}$ at room temperature before incubation with primary antibodies (1:50 dilution in PBS containing $0.1 \%$ Tween and $0.3 \%$ BSA). We used the following primary antibodies: anti-rattin (HNr; rabbit-polyclonal, PA1-46257; Thermo Fisher Scientific), anti-ionized calcium-binding adaptor molecular 1 (Iba1; rabbitpolyclonal, 019-19741; Wako Life Science), and anti-GFAP (mousemonoclonal, G7171, Sigma Millipore). Secondary antibodies (1:100 dilution in PBS containing $0.1 \%$ Tween and $0.3 \%$ BSA) included the following: goat anti-rabbit IgG (AlexaFluor-488 or 546, Thermo Fisher Scientific) and goat anti-mouse IgG (AlexaFluor-488, Thermo Fisher Scientific). Brain images were captured using an LSM 800 confocal microscope with Airyscan high-resolution detectors (Carl Zeiss). For immunofluorescent analysis of primary rat astrocytes, we used antilysosomal-associated membrane protein 1 (LAMP1; 1:250 dilution, rabbit-polyclonal, ab24170; Abcam) and goat anti-rabbit IgG (1:500 di- 
lution, AlexaFluor-488, Thermo Fisher Scientific). The procedure for immunofluorescence was published previously (Jung et al., 2015).

Primary cerebral cortical glial cell cultures. Rat astrocyte and microglia cultures were generated using embryonic day 17 or 18 brains, as we previously described (X. Zhao et al., 2007b, 2017). Briefly, mixed cells from brain cortices were seeded in poly-D-lysine-coated $75 \mathrm{~cm}^{2}$ flasks and cultured in DMEM containing $10 \%$ FBS, penicillin $(50 \mathrm{U} / \mathrm{ml})$, and streptomycin $(50 \mu \mathrm{g} / \mathrm{ml})$ for $10-14 \mathrm{~d}$. Once cells were confluent in the flask, adherent microglia were harvested using a slight shaking method $\left(37^{\circ} \mathrm{C}\right.$ at $220 \mathrm{rpm}$ for $\left.30-60 \mathrm{~min}\right)$. Harvested microglia were replated onto poly-D-lysine-coated plates with DMEM containing $10 \%$ FBS, penicillin $(50 \mathrm{U} / \mathrm{ml})$, and streptomycin $(50 \mu \mathrm{g} / \mathrm{ml})$. After microglia harvest, the cocultures were trypsinized with $0.25 \%$ Trypsin-EDTA to collect the mixed astroglial cells attached to the bottom of the flask. These were replated onto $75 \mathrm{~cm}^{2}$ flasks and cultured in DMEM containing 10\% FBS, penicillin $(50 \mathrm{U} / \mathrm{ml})$, and streptomycin $(50 \mu \mathrm{g} / \mathrm{ml})$. Once the cells were slightly attached to the bottom (4-6 h after replating), the flask was transferred into a $37^{\circ} \mathrm{C}$ shaking incubator and continuously shaken $(220$ $\mathrm{rpm}$ ) overnight to remove weakly attached cells. The medium containing floating cells was discarded the next day, and new fresh culture medium was added to the strongly attached astrocytes.

FACS analysis for Mt. FACS analysis to detect secreted Mt from cultured astrocytes was performed on a Cytoflex S cytometer (Beckman Coulter). The data were analyzed with FloJo version 10. Rat cortical astrocytes were incubated with Mitotracker Red CMXRos (M7512, Thermo Fisher Scientific) for $30 \mathrm{~min}$ to label intracellular Mt. After extensive washing, the astrocytes received fresh culture medium and were incubated for $24 \mathrm{~h}$ to allow secreted Mt accumulation. The culture medium was collected and briefly spun down at $1000 \mathrm{rpm}$ for $5 \mathrm{~min}$ to remove cellular debris. Half of the supernatant was saved as astrocyteconditioned medium (ACM), and the other half was filtered through a column with $0.22 \mu \mathrm{m}$ pores to remove Mt particles (Filtered-ACM) as previously described (Hayakawa et al., 2016). Unstained ACM (without Mitotracker treatment) was used as a negative control for proper gating.

Transmission electron microscope (TEM) analysis. To probe ACM for extracellularly released $\mathrm{Mt}$, collected ACM was subjected it to $4020 \times g$ centrifugation for $30 \mathrm{~min}$ at $4^{\circ} \mathrm{C}$ to produce a pellet containing Mt. The pellet was resuspended, transferred to a $1.5 \mathrm{ml}$ Eppendorf tube, and further spun down at $13,400 \times g$ for $15 \mathrm{~min}$ at $4^{\circ} \mathrm{C}$. The final Mtcontaining pellet was washed with PBS and fixed with $2 \%$ glutaraldehyde in $0.1 \mathrm{M}$ sodium cacodylate buffer, $\mathrm{pH}$ 7.4. Fixed samples were washed in $0.1 \mathrm{M}$ sodium cacodylate buffer and treated with $0.1 \%$ cacodylatebuffered tannic acid, postfixed with $1 \%$ buffered osmium, and stained with $1 \%$ uranyl acetate. The samples were dehydrated in increasing concentrations of ethanol and embedded in LX-112 medium. The samples were polymerized in a $60^{\circ} \mathrm{C}$ oven for $\sim 3 \mathrm{~d}$. Ultrathin sections were cut using a microtome (Leica Microsystems), stained with uranyl acetate and lead citrate in a Leica Microsystems EM Stainer, and examined in a JEM 1010 transmission electron microscope (JEOL) at an accelerating voltage of $80 \mathrm{kV}$. Digital images were obtained using an AMT Imaging System (Advanced Microscopy Techniques). Sample processing and TEM evaluation were conducted at the High Resolution Electron Microscopy Facility of the University of Texas M.D. Anderson Cancer Center.

Red blood cell (RBC) isolation. RBCs from rat peripheral blood were isolated using column density gradient centrifugation (BD Vacutainer CPT, BD Biosciences) as we described previously (X. Zhao et al., 2007b).

Phagocytosis in vitro. To measure microglial phagocytic activity, in vitro phagocytosis assays were performed as described previously (X. Zhao et al., 2007b). Briefly, RBCs were added to the cultured microglia and incubated for $2 \mathrm{~h}$. Then, the culture plate was briefly centrifuged to remove unphagocytosed RBCs. Microglia containing phagocytosed RBCs were fixed with $4 \%$ PFA and stained with anti-CD11b (mouse monoclonal, MCA275G; Bio-Rad) and anti-rat RBC (rabbit polyclonal, 20R-RR012; Fitzgerald Industries) antibodies to visualize internalized RBCs. The number of engulfed RBCs in microglia was used as a phagocytosis index.

Small interfering RNA (siRNA) transfection. siRNA probes targeting $\mathrm{HNr}$ (GGTTCAACTGTCTCTTACT and GGGATAACAGCGCAATCCT) were obtained from Sigma Millipore. The nontargeting, negative control, scrambled siRNA (SIC001) was obtained from Sigma Millipore. Primary rat cortical astrocytes were seeded on 6 -well plates $\left(2 \times 10^{6}\right.$ cell/well $)$ and transfected with each siRNA $(10 \mathrm{nmol} / \mathrm{L})$ using HiPerFect siRNA transfection reagent (301704; QIAGEN) for $24 \mathrm{~h}$ following the manufacturer's instructions. The ACM for transfer onto microglia was collected as described above.

$R T-P C R$ analysis. Total RNA was extracted from rat primary microglia or the contralateral and ipsilateral ICH-affected mouse brain using the RNeasy Plus Mini Kit (74134, QIAGEN). The cDNAs were generated using amfiRivert Plantinum ONE cDNA Synthesis Master Mix (R6100, GenDEPOT). The amfiSure qGreen Q-PCR Master Mix without ROX (Q5600, GenDEPOT) was used for SYBR real-time RT-PCR analysis. The following sets of primers $\left(5^{\prime}-3^{\prime}\right)$ were used for experiments: $\mathrm{HNr}$, TTAGGGACTAGAATGAATGG and GGAGCTTCAATTTACTAGTT; rat PPAR $\gamma$, ACCAACTTCGGAATCAGCTC and AGGCTCTACTTT GATCGCAC; rat lipoprotein lipase (LPL), CAGCTGGGCCTAACTTT GAG and TGCTGGGGTTTTCTTCATTC; rat catalase, ATGCAAAGGG AGCAGGTG and AATGGGAAGGTTTCTGCC; rat IL-10, TCCTT GGAAAACCTCGTTTG and GCTTTCGAGACTGGAAGTGG; rat IL$1 \beta$, CAGGAAGGCAGTGTCACTCA and GGGATTTTGTCGTTGCTT GT; rat CD68, AATGTGTCCTTCCCACAAGC and AGAGGGGCTGGT AGGTTGAT; rat GAPDH, AGACAGCCGCATCTTCTTGT and TAC TCAGCACCAGCATCACC; and mouse GAPDH, TGTTCCTACCCC CAATGTGT and TGTGAGGGAGATGCTCAGTG.

Western blot. Protein samples were prepared from whole-cell lysates as previously described (Jung et al., 2009, 2015). Briefly, protein samples were separated on $4 \%-20 \%$ gradient Tris-glycine SDS gels (EC6021, Thermo Fisher Scientific) followed by electrophoretic transfer onto nitrocellulose membranes using an iBlot2 Dry Blotting System (IB21001, Thermo Fisher Scientific). Antibodies against Mn-SOD (1:1000 dilution; rabbit polyclonal, ADI-SOD-110-D; Enzo Life Science), PPAR $\gamma$ (1:1000 dilution; rabbit polyclonal, sc-7196; Santa Cruz Biotechnology), and $\beta$-tubulin (1:5000 dilution; mouse monoclonal, T8328, Sigma Millipore) were used for immunoblotting. HRP-conjugated IgG and an enhanced chemiluminescent substrate (Pierce ECL kit 32106, Thermo Fisher Scientific) were used to visualize protein bands on $\mathrm{x}$-ray films. The band intensity was analyzed by ImageJ program (U.S. National Institutes of Health) and normalized by intensity of control band ( $\beta$-tubulin).

Dot blot. To detect HNr that is released into media, we collected ACM in $75 \mathrm{~cm}^{2}$ tissue culture flasks. The astrocytes were incubated with 5-6 $\mathrm{ml}$ of serum-free DMEM for $24 \mathrm{~h}$ before concentrating ACM to $100 \mu \mathrm{l}$ to reach a concentration of $30 \mu \mathrm{g}$ of proteins in $10 \mu \mathrm{l}$ of loading volume for dot blotting. To concentrate released $\mathrm{HNr}$, collected ACM were centrifuged with Vivaspin 20 Centrifuge Concentrators (VS2091, 3000 Dalton Max Molecular Weight Cut Off, PES membrane; Sartorius). Dot blot analysis using a Bio-Dot SF Microfiltration Apparatus (1706545, BioRad) was used to assess $\mathrm{HNr}$ concentration. Briefly, ACM protein was deposited onto nitrocellulose membranes in the dot blot apparatus. The membranes were blocked with 5\% BSA in Tris-buffered saline with Tween and then incubated with anti-rattin $(\mathrm{HNr})$ antibody (1:1000 dilution; rabbit-polyclonal, PA1-46257, Thermo Fisher Scientific). Secondary HRP-conjugated $\operatorname{IgG}$ and an enhanced chemiluminescent substrate were used to visualize $\mathrm{HNr}$ as described above. In addition, an Odyssey CLx imaging system (LI-COR) using IRDye 800CW goat antirabbit IgG secondary antibody (1:5000 dilution; 925-32211, LI-COR) or IRDye $800 \mathrm{CW}$ Goat anti-Mouse IgG secondary antibody (1:5000 dilution; 925-32210, LI-COR) was used to visualize $\mathrm{HNr}$ or $\beta$-tubulin (see Figure $9 B$ ). The immunointensity of each of the dots was analyzed by ImageJ program and normalized to intensity of $\beta$-tubulin, control.

Experimental design and statistical analyses. For all the in vivo animal studies, animals were randomly divided for treatments and ICH surgery. The sample size calculation was based on our past experience with mouse ICH model (X. Zhao et al., 2007a,b, 2009b, 2017), regarding reproducibility in the amount of damage, and power analysis using STATMATE 3 software (a significant difference at a power of $90 \%$, estimated SD of mean at $\sim 0.1-0.2$, and expected difference between groups at $10 \%-$ $20 \%$ ) to obtain the minimal number of animals for statistical significance. For animal behavior tests, two-way ANOVA followed by the Fisher LSD method was used to compare NDS between two groups at 

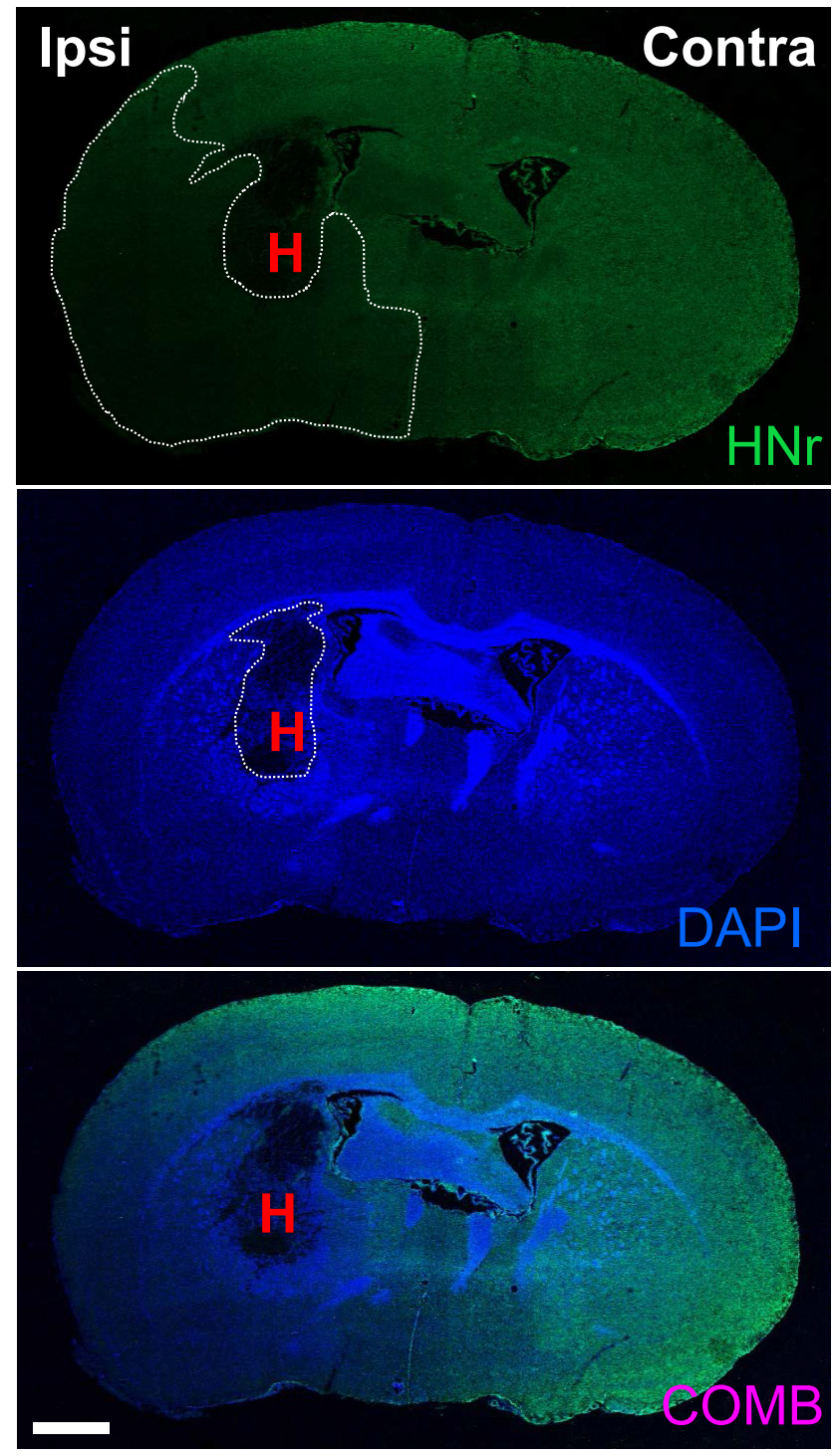

Figure 1. ICH reduces HN expression in the affected hemisphere. HNr immunoreactivity is reduced in the $\mathrm{ICH}$-affected forebrain compared with contralateral hemisphere at $24 \mathrm{~h}$ after $\mathrm{ICH}$. Representative image reconstructing a coronal section of the ICH mouse brain under fluorescence microscope $(10 \times)$ showing the HN signal detected using anti-HNr (Rattin; a rat homolog of HN) antibody. Scale bar, $1000 \mu \mathrm{m}$. DAPI was used to visualize nuclei. H, Hematoma; Contra, contralateral; Ipsi, ipsilateral; COMB, combined.

different time points. Data are presented as mean \pm SEM. Most in vitro studies were analyzed by one-way ANOVA followed by the Fisher's Least Significant Difference (LSD) method, or followed by Kruskal-Wallis test or Bonferroni test between experimental groups. Paired or unpaired $t$ tests were used to compare two groups. Statistically significant differences between mean values were considered only at $p<0.05$. Prism 7.04 software (GraphPad Software) was used for statistical analysis. The detailed results of statistical analyses, including the number or size of each experiment, are listed in each figure legend.

\section{Results}

ICH reduces $\mathrm{HN}$ level in the affected brain hemisphere

Reduced HN levels adversely affect many pathological processes, longevity, and aging (Muzumdar et al., 2009; Voigt and Jelinek, 2016). It is therefore relevant to understand how cerebrovascular events, such as ICH, affect $\mathrm{HN}$ and influence disease progression. Hence, we evaluated mRNA and protein levels of $\mathrm{HN}$ in the ICHaffected brain. Using SYBR real-time RT-PCR and brains har-
A

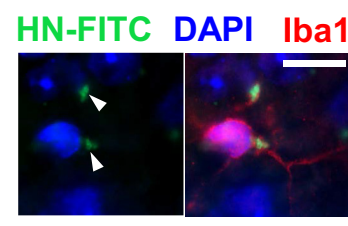

Ex 2

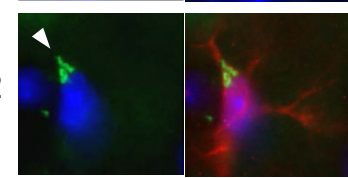

B

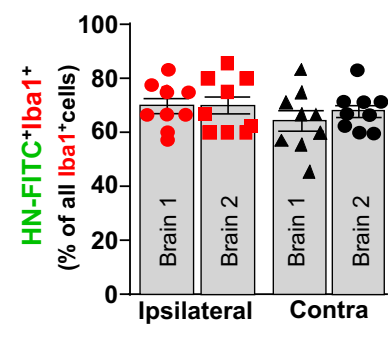

Figure 2. Intravenously administered $\mathrm{HN}$ is found in microglia in the mouse brain. $A, \mathrm{HN}$ FITC ( $1 \mu \mathrm{g} / \mathrm{g}$ in PBS) was injected via the femoral vein $24 \mathrm{~h}$ after ICH onset. After another $24 \mathrm{~h}$, confocal microscopy was used to detect HN-FITC (green, white arrowheads) in the ICH-affected brain (ipsilateral) and their colocalization with Iba ${ }^{+}$cells (red). Representative examples of two randomly selected HN-FITC-positive cells are shown. Nuclei were stained with DAPI (blue). Scale bar, $10 \mu \mathrm{m}$. B, Quantitative bar graph (mean \pm SEM) showing the percentage of HNFITC/Iba1 double-positive cells among all the lba ${ }^{+}$cells, as assessed in nine or 10 randomly selected perihematoma (ipsilateral) and homologous contralateral locations (Contra) in two independently analyzed mouse brains.

vested at $24 \mathrm{~h}$ after $\mathrm{ICH}$, we found a robust $\sim 50 \%$ reduction in $\mathrm{HNr}$ mRNA levels in the ICH-affected forebrain compared with the homologous contralateral hemisphere (mRNA ratio of contralateral vs ipsilateral is $1: 0.574, n=13$, two-tailed paired $t$ test, $p=0.000198, t=5.27)$. We also observed a profound loss of $\mathrm{HNr}$ immunoreactivity throughout the ICH-affected hemisphere compared with the contralateral brain (Fig. 1). Considering the localized nature of the ICH lesion (5\%-10\% of the hemisphere), the extent of $\mathrm{HNr}$ immunoreactivity loss was widespread in the ipsilateral brain.

\section{$\mathrm{HN}$ reduces neurological deficits and improves hematoma clearance in ICH mice}

Since ICH dramatically decreased $\mathrm{HNr}$ in the brain, we examined whether $\mathrm{HN}$ administration could affect the severity of $\mathrm{ICH}$ induced injury. First, we intravenously injected mice with $\mathrm{HN}$ FITC $24 \mathrm{~h}$ after ICH. Confocal microscopy was performed to inspect brain tissue for the presence of green fluorescence. We established that Iba1-positive cells (e.g., microglia and macrophages) are one of the prominent cell targets for HN-FITC, based on colocalization of fluorescence signals (Fig. 2A). By probing cells in randomly selected locations around hematoma location and in homolog locations of the contralateral hemisphere, we found that $\sim 67 \%$ of all $\mathrm{Iba}^{+}{ }^{+}$cells were also positive for FITC (double-positive) (Fig. 2B), indicating an overall abundant internalization of $\mathrm{HN}$ to the brain and microglia.

Next, we used a mouse ICH model to determine whether HN has a therapeutic effect in ICH-induced brain injury. Specifically, we tested whether HN could reduce neurological deficits (NDS; measured with corner, postural flexing, or foot-fault testing) resulting from ICH injury. $\mathrm{HN}$ was injected once a day $(1 \mu \mathrm{g} / \mathrm{g}$ in PBS, i.p., starting $1 \mathrm{~h}$ after ICH) for a total of 7 administrations. This significantly reduced NDS in HN-treated mice (Fig. $3 A, B$ ). In addition to the functional benefit, $\mathrm{HN}$ accelerated hematoma clearance, a process that is mediated by phagocytic cells, including microglia/macrophages. This was established by reduced hemoglobin content (a major hematoma component) $7 \mathrm{~d}$ after ICH in HN-treated mice compared with vehicle-treated mice (Fig. $3 C)$. In a parallel experiment, we assessed whether the therapeutic effect of HN could also be achieved with intranasal HN. We extended the therapeutic window for $\mathrm{HN}$ to $3 \mathrm{~h}$ after $\mathrm{ICH}$, which is 


\section{Intraperitoneal administration}
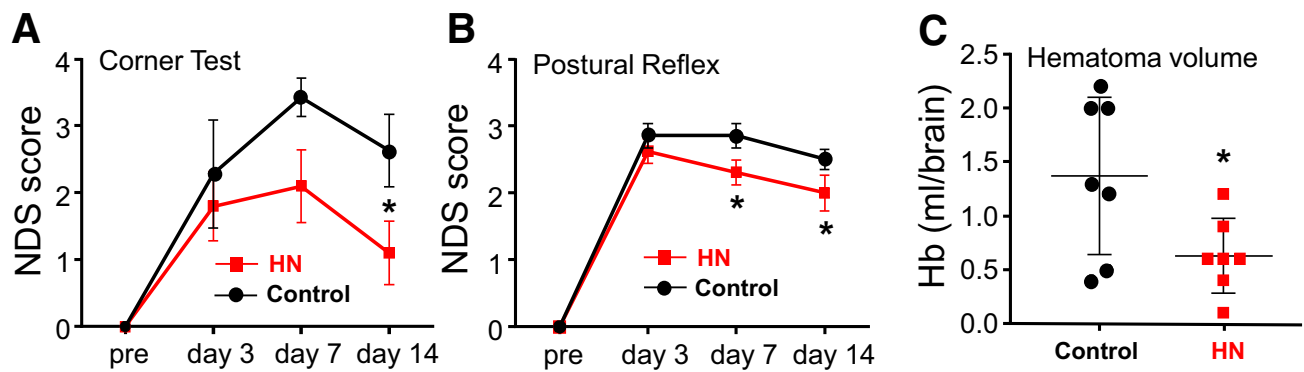

Intranasal administration

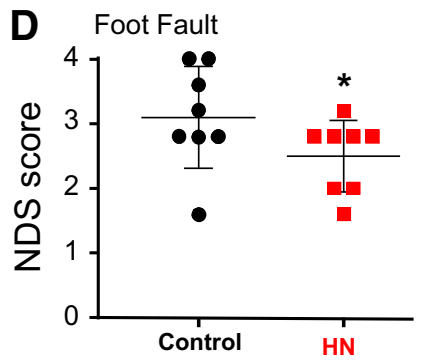

Figure 3. HN treatment reduces neurological deficits and improves hematoma clearance in an ICH mouse model, suggesting a role of phagocytic cells. NDSs were calculated from corner, postural flexing, or foot-fault testing in mice treated either with $\mathrm{HN}$ given intraperitoneally $(1 \mu \mathrm{g} / \mathrm{g})(\boldsymbol{A}, \boldsymbol{B})$ or intranasally $(1 \mu \mathrm{g} / \mathrm{g})(\boldsymbol{D})$ starting $1 \mathrm{~h}(\boldsymbol{A}, \boldsymbol{B})$ or $3 \mathrm{~h}$ (D) after ICH onset. PBS was used as a vehicle control. C, Hemoglobin content as an index of hematoma clearance in ICH-affected brains on day 7 after ICH in mice treated with intraperitoneal HN or PBS. Data are mean \pm SEM. $A$, Two-way ANOVA/Fisher's LSD test, $n=8$ per group, ${ }^{*} p<0.05(p=0.0271), t$ values $(t=2.275)$. $\boldsymbol{B}$, Two-way ANOVA/Fisher's LSD test, $n=8$ per group, ${ }^{*} p<0.05(p=0.0299), t$ values $(t=2.232)$ at day $7 ;{ }^{*} p<0.05(p=0.0455)$, $t$ values $(t=2.049)$ at day 14. C, Two-tailed unpaired $t$ test, $n=7$ per group, ${ }^{*} p<0.05(p=0.0322)$, $t$ values $(t=2.423)$. D, Two-way ANOVA/Fisher's LSD test, $n=8$ per group, ${ }^{*} p<0.05(p=0.0195)$, $t$ values $(t=2.479)$ at day 3 .

a more clinically relevant protocol. Mice were treated with $\mathrm{HN}$ at $1 \mu \mathrm{g} / \mathrm{g}$ in PBS for a total of 7 administrations. Similar to the intraperitoneal route, intranasal $\mathrm{HN}$ reduced NDS (Fig. 3D).

Astrocytes in culture secrete $\mathrm{HN}$, and $\mathrm{HN}$ is present in astrocytes in the brain $\mathrm{HN}$ is a peptide encoded by Mt DNA (Yen et al., 2013). Once translated, $\mathrm{HN}$ is primarily confined to $\mathrm{Mt}$, but it can also be released to the cytoplasm (Yen et al., 2013). Mt themselves are the major storage site for HN. A recent study demonstrated that astrocytes release $\mathrm{Mt}$, and other cell types can uptake these Mt (Hayakawa et al., 2016). This suggests that the biological effects of HN could be mediated through its release to the extracellular space and/or cell-to-cell transfer via astrocyte-secreted Mt.

To test this hypothesis, we examined whether $\mathrm{HN}$ is produced and secreted by primary rat astrocytes in culture. ACM was collected in an established culture system. Using dot blots and an antibody against $\mathrm{HNr}$, we demonstrated that cultured astrocytes indeed produce and secrete $\mathrm{HNr}$ (Fig. 4A). Specifically, we collected ACMs from five rat astrocyte preps, and performed dot immunoblots to quantitate secreted HNr. Compared with DMEM, we found $\sim 14$-fold increase in $\mathrm{HNr}$ level in ACM (DMEM vs $\mathrm{ACM}=1: 14.506, n=5$, two-tailed unpaired $t$ test, $p=0.0075$, $t=3.547)$. In addition, immunohistochemistry experiments

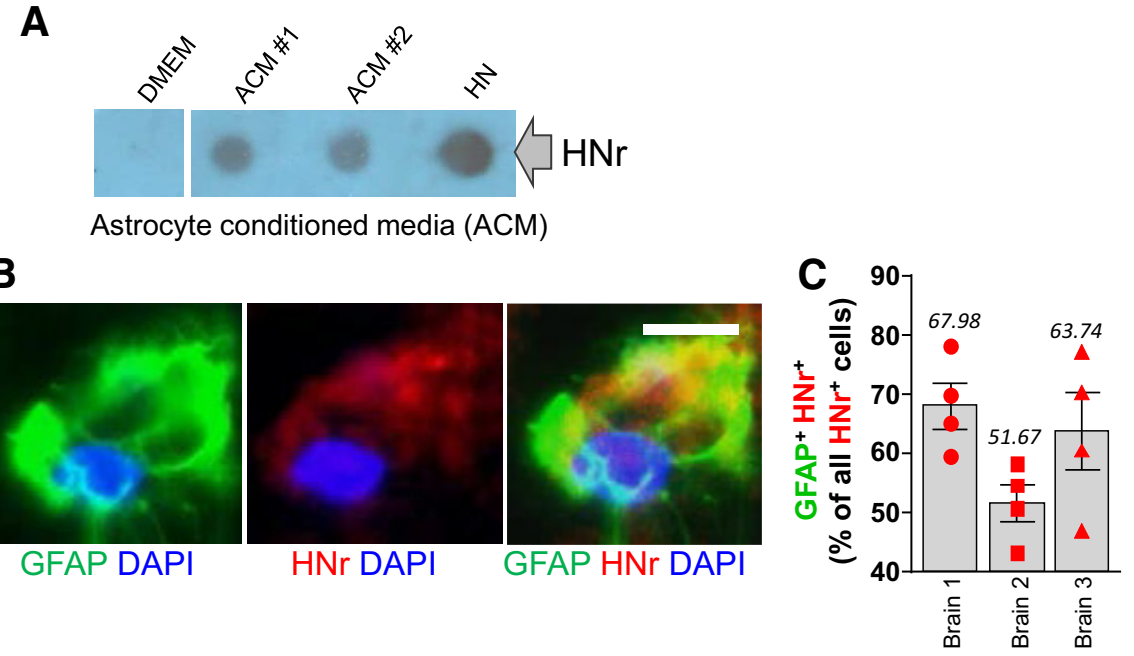

Figure 4. Astrocytes in culture secrete $H N$, and $H N$ is present in astrocytes in the brain. $A$, Rat ACM was concentrated using Vivaspin and spotted onto nitrocellulose membranes. HN (2 $\mu \mathrm{l}$ from $10 \mathrm{~mm}$ HN stock) and DMEM were used as positive and negative controls, respectively. Image represents two different ACM samples from each culture that were spotted. Extracellularly released HN was detected using anti-HNr (a rat homolog of HN) antibody. $\boldsymbol{B}$, A strong HNr immunofluorescence signal (red) was observed in GFAP ${ }^{+}$(green) rat astrocytes. Confocal image. Scale bar, $6.25 \mu \mathrm{m}$. C, Quantitative bar graph (mean \pm SEM) showing the percentage of HNr/GFAP double-positive cells among all the $\mathrm{HNr}^{+}$cells, as assessed in four randomly selected locations in three independently analyzed mouse brains.

confirmed strong presence of $\mathrm{HNr}$ immunoreactivity in GFAP ${ }^{+}$ cells in the inspected cerebral cortex of the normal rat brain (Fig. $4 B)$. We quantified the numbers of $\mathrm{GFAP}^{+} / \mathrm{HNr}^{+}$doublepositive cells in the randomly selected areas of cerebral cortex of 3 naive rats. Approximately $61 \%$ of $\mathrm{HNr}^{+}$cells showed GFAPpositive (Fig. 4C), confirming that astrocytes could be an important source of $\mathrm{HN}$. 

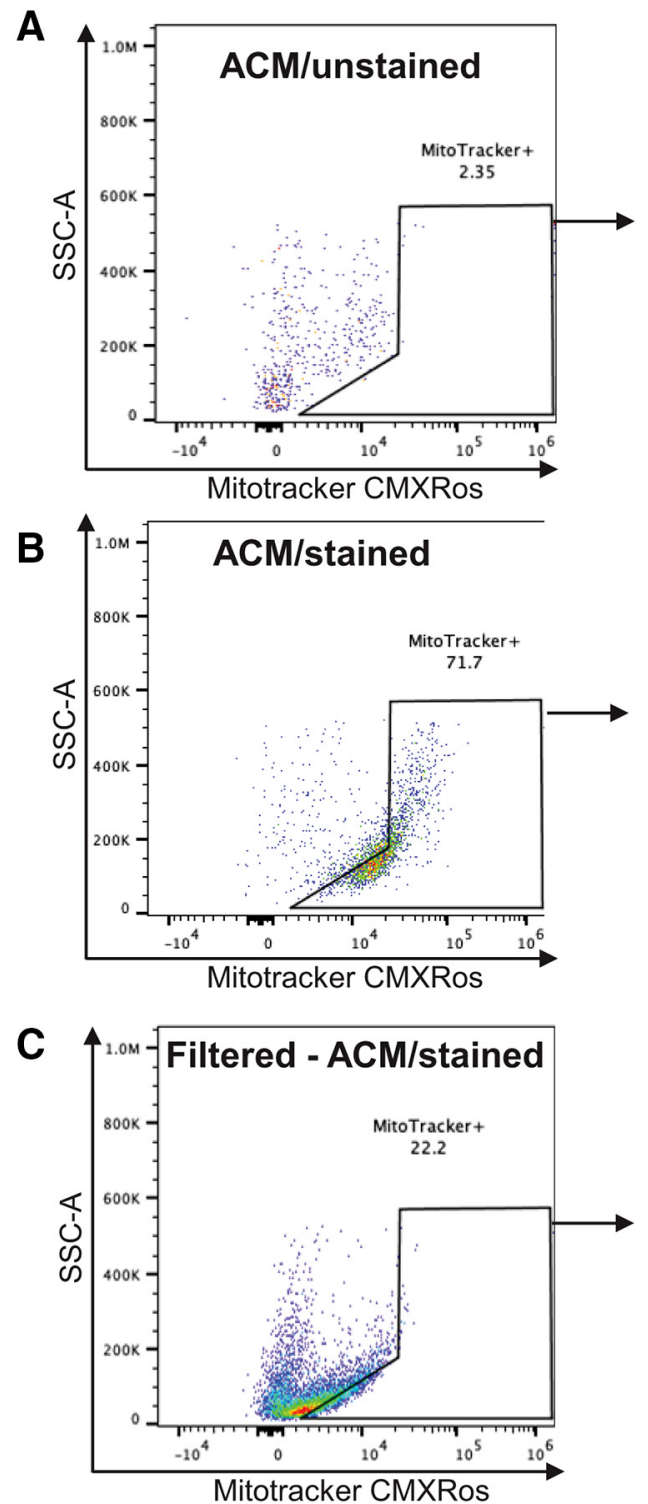
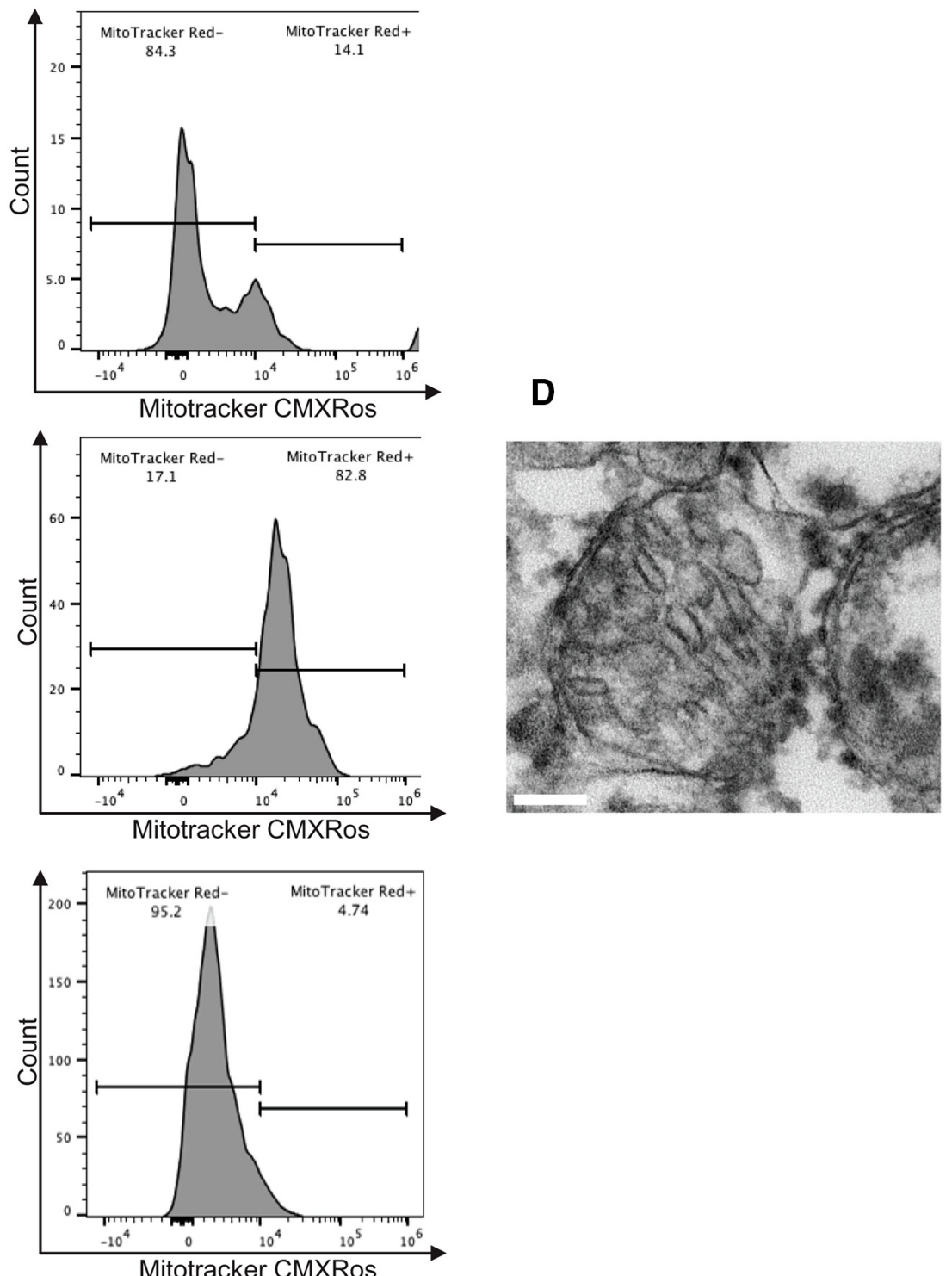

Figure 5. Mt are released by astrocytes in vitro. Rat cortical astrocytes were incubated for 30 min with Mitotracker/Red/CMXRos to label Mt. After extensive washing, astrocytes were incubated with fresh culture medium for $24 \mathrm{~h}$ to accumulate Mt in the media. ACM was collected and briefly spun down to remove cellular debris and assessed for the presence of Mitotracker-positive Mt using FACS analysis. $\boldsymbol{A}$, Negative control, ACM from astrocytes that were not stained with Mitotracker/Red/CMXRos. $\boldsymbol{B}$, ACM collected from astrocytes stained with Mitotracker/Red/CMXRos. $\boldsymbol{C}$, ACM collected as in $\boldsymbol{B}$, but filtered through a $0.22 \mu \mathrm{m}$ pore-size column to remove Mt (Filtered-ACM). FACS analysis data for the above samples. $\boldsymbol{D}$, An example of Mt found in ACM as detected using TEM. Scale bar, $100 \mathrm{~nm}$.

\section{Astrocyte-secreted Mt incorporate into microglia and subsequently increase $\mathrm{HNr}$ levels}

To confirm that astrocytes release $\mathrm{Mt}$, primary astrocyte cultures were incubated with or without Mitotracker Red CMXRos to fluorescently label Mt for FACS analysis of ACM (Fig. 5A,B). Mt were abundant in the ACM, suggesting that astrocytes indeed secrete large quantities of Mt (Fig. 5B). Hayakawa et al. (2016) used qNano analysis to show that astrocyte-released Mt range in size from 300 to $1100 \mathrm{~nm}$ and, as such, could be removed from conditioned media with a $0.22 \mu \mathrm{m}$ filter. Quantification of MitoTracker-positive particles revealed that ACM filtration through a $0.22 \mu \mathrm{m}$ filter removed $78 \%$ of Mt (Fig. $5 C$ ). TEM confirmed the presence of Mt in ACM (Fig. 5D).

Next, we tested whether Mt released by astrocytes can enter microglia. We labeled Mt in rat astrocytes with MitoTracker/ CMX/Ros (Fig. 6A,B, red, white arrowheads) and collected ACM-containing MitoTracker-positive Mt for transfer into rat primary microglia cultures (Fig. 6A). This approach revealed that astrocytic Mt effectively integrated into microglia (Fig. 6C,D). Time-lapse imaging confirmed Mt transfer into microglia. The images of two representative microglial cells (Fig. 6C, colorcoded blue and red) taken before Mt transfer ( $0 \mathrm{~min}$, top), at the indicated times after ACM addition between $15 \mathrm{~min}$ and $11 \mathrm{~h} 11$ min (Fig. 6C, middle), and at $14 \mathrm{~h}$ at the end of the recording (Fig. $6 C$, bottom) show the dynamics of microglial Mt accumulation. All the microglial cells showed the presence of MitoTracker redpositive Mt (Fig. 6-1, available at https://doi.org/10.1523/ JNEUROSCI.2212-19.2020.f6-1). Upon adding ACM, Mt incorporate into the cultured microglia almost immediately and continue to accumulate for hours. It is essential to note that $\mathrm{Mt}$ that entered microglia remained intact for at least $72 \mathrm{~h}$ (Fig. 6D), suggesting that these $\mathrm{Mt}$ are not just a target of phagocytosis and consequent degradation. To strengthen this claim, we quantified the numbers of LAMP- $1^{+} /$MitoTracker $^{+}$(double-positive) par- 


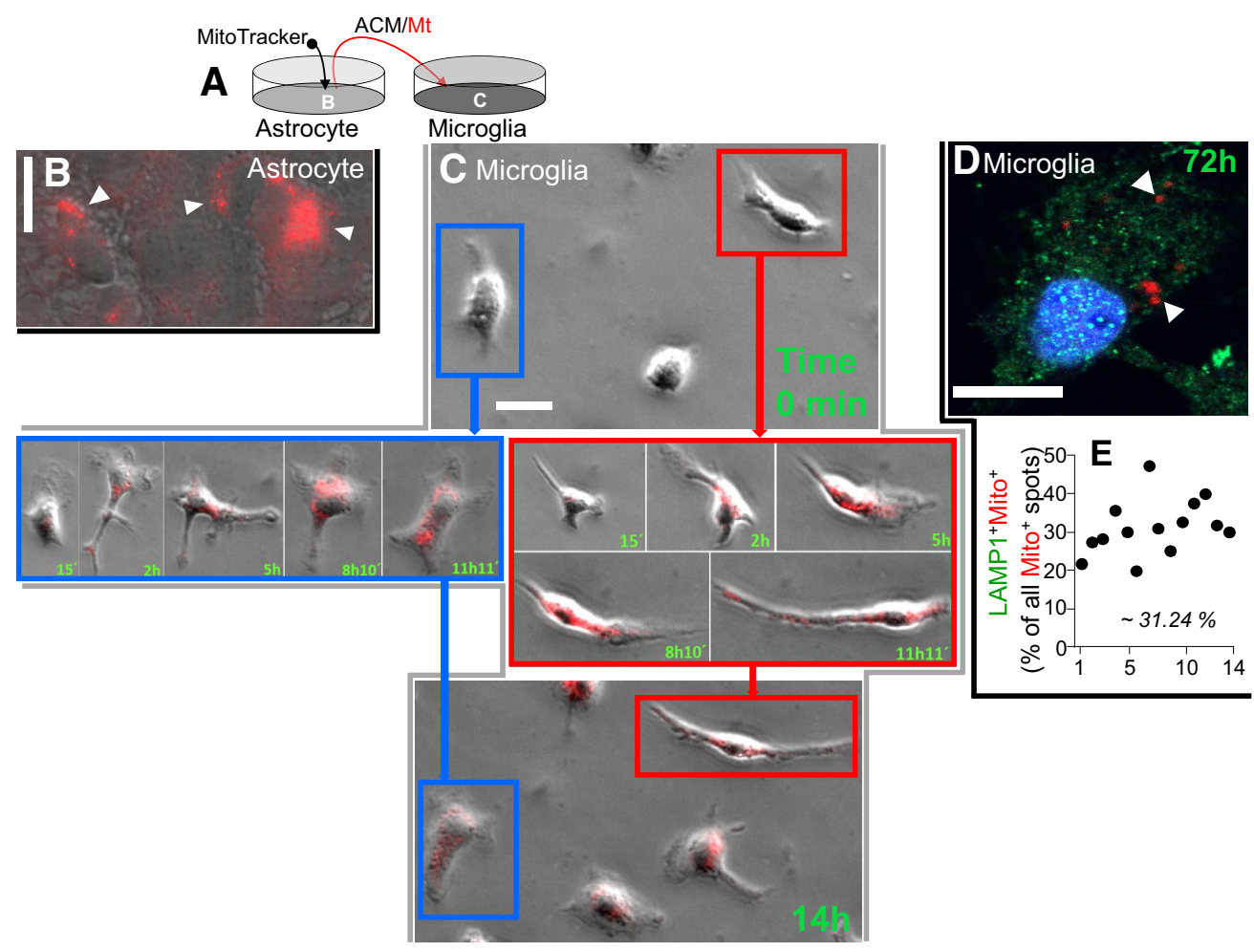

Figure 6. Astrocyte-secreted Mt incorporate into microglia and elevate intracellular HN levels. A, Astrocytes in culture were treated with MitoTracker/CMX/Ros to red-fluorescently label Mt. After extensive washing to remove MitoTracker and additional $24 \mathrm{~h}$ incubation to accumulate Mt in the media, the media containing red-labeled astrocytic Mt were transferred to rat primary microglia in culture. $\boldsymbol{B}$, Representative confocal image of rat astrocytes in culture treated with MitoTracker/CMX/Ros to label Mt (red puncta, white arrowheads). Scale bar, $20 \mu \mathrm{m}$. C, Microglia taking up astrocytic Mt. Time-lapse images were collected to document Mt (red) transfer into microglia over a $14 \mathrm{~h}$ period. Images of two representative microglial cells (color-coded as blue and red) before Mt transfer ( 0 min, top) and at the indicated times ( $15 \mathrm{~min}$ to $11 \mathrm{~h}$, middle; and at $14 \mathrm{~h}$, bottom). Scale bar, $20 \mu \mathrm{m}$. At the end of the recording, all the microglial cells showed the presence of MitoTracker (Figure 6-1, available at https://doi.org/10.1523/JNEUROSCI.2212-19.2020.f6-1). D, Confocal image of microglial cells containing astrocytic Mt (MitoTracker red ${ }^{+}$puncta). These astrocyte-derived $\mathrm{Mt}$ (red, white arrowheads) are retained in microglia for at least $72 \mathrm{~h}$ after internalization. Most of the Mt (Mito ${ }^{+}$red puncta) did not colocalize with the LAMP-1 (green puncta), suggesting that Mt are not merely undergoing lysosomal degradation. Scale bar, $10 \mu \mathrm{m}$. E, Quantitative data showing the percent of LAMP-1/MitoTracker double-positive puncta among all the MitoTracker red ${ }^{+}$puncta, as determined for 14 randomly selected microglial cells.

ticles within 14 randomly selected microglia (Fig. 6E). We established that $31.24 \%$ of MitoTracker ${ }^{+}$speckles were also LAMP$1^{+}$. This indicates that most of the internalized $\mathrm{Mt}(\sim 69 \%)$ did not colocalize with the phagosome marker LAMP-1 at $72 \mathrm{~h}$.

Since $\mathrm{Mt}$ are the main source of $\mathrm{HN}$, astrocyte-released $\mathrm{Mt}$ could transport $\mathrm{HN}$ in the form of DNA, mRNA, and/or protein. We therefore tested whether astrocytic Mt accumulated in microglia could elevate HN content in these cells. ACM or filtered ACM (mdACM; Mt-depleted ACM) (Fig. 7A,B) was added to rat microglia and incubated for $24 \mathrm{~h}$. Indeed, incorporation of astrocytic Mt into microglia significantly increased levels of $\mathrm{HNr}$ mRNA (Fig. 7A) and protein (Fig. 7B). When Mt were removed by passage through a $0.22 \mu \mathrm{m}$ filter, there was no increase in microglial $\mathrm{HNr}$ (Fig. $7 A, B$ ). These data suggest that astrocytic $\mathrm{Mt}$ transfer HNr to microglia, which increases HNr levels.

\section{HN or Mt upregulate PPAR $\gamma / \mathrm{Mn}-\mathrm{SOD}$ and other prototypic PPAR $\gamma$ target genes in microglia}

Following ICH, the transcription factor PPAR $\gamma$ and its target genes play central roles in promoting a "reparative" microglia phenotype that is protrophic and phagocytic but anti-inflammatory phenotype (Ricote et al., 1999; X. Zhao et al., 2007b; Yi et al., 2008). Mn-SOD (a transcriptional target of PPAR $\gamma$ ), an enzyme exclusively confined to $\mathrm{Mt}$, plays an essential role by maintaining the oxidative balance necessary to maintain this "reparative" microglial phenotype. Hence, we tested whether microglial PPAR $\gamma$ and Mn-SOD are upregulated in response to $\mathrm{HN}$ or
A

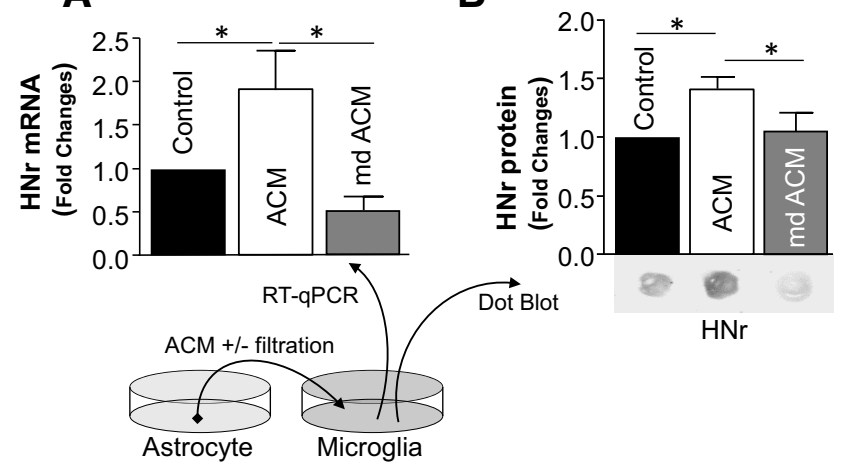

Figure 7. ACM containing Mt, but not Mt-depleted ACM, induced microglial HN synthesis. ACM or Mt-depleted ACM (mdACM, filtered ACM through a $0.22 \mu \mathrm{m}$ pore-size column to remove Mt) was transferred onto cultured microglia. After $24 \mathrm{~h}$, HNr mRNA levels $(\boldsymbol{A})$ were assessed by SYBR real-time RT-PCR. Data are mean \pm SEM; $n=5$. One-way ANOVA/Fisher's LSD test: ${ }^{*} p<0.05$ ( $p=0.0380$, Control vs ACM), $t$ values $(t=2.358) ;{ }^{*} p<0.01$ ( $p=0.0062$, $A C M$ vs mdACM), $t$ values $(t=3.373)$. $B$, HNr protein levels were assessed by immune dot blot. Data are mean \pm SEM; $n=3$. One-way ANOVA/Fisher's LSD test: ${ }^{*} p<0.05(p=0.0366$, Control vs ACM), $t$ values $(t=2.678) ;{ }^{*} p<0.05(p=0.0479, A C M$ vs mdACM), $t$ values $(t=$ 2.478).

after astrocytic Mt transfer (another source of intra-Mt $\mathrm{HNr}$ ). ACM (Fig. $8 A, B$ ) or mdACM (Fig. $8 B$ ) was incubated with rat microglia for $24 \mathrm{~h}$. In a parallel experiment, $\mathrm{HN}$ alone (Fig. $8 \mathrm{~A}, \mathrm{~B}$ ) or in the presence of $\mathrm{HN}$-neutralizing antibody (Fig. $8 B$ ) was 

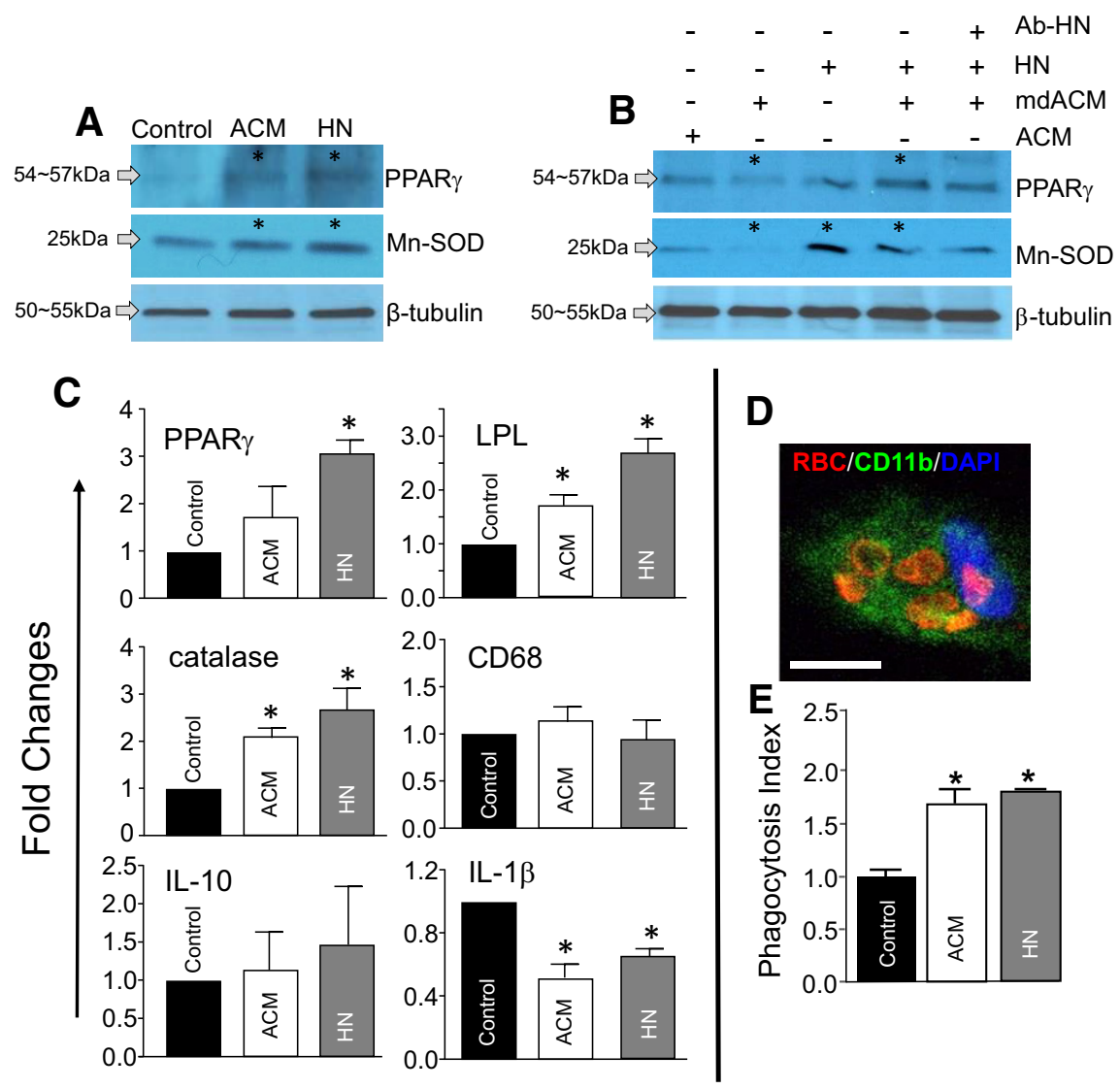

Figure 8. HN or ACM upregulates PPAR $\gamma / \mathrm{Mn}-\mathrm{SOD}$ and other prototypic PPAR $\gamma$ target genes in microglia, and enhances microglial phagocytic activity in an in vitro hematoma clearance model. $A$, Western blots determine microglial induction of PPAR $\gamma$ and $\mathrm{Mn}$-SOD at $24 \mathrm{~h}$ after exposure to ACM or HN $(20 \mu \mathrm{m})$. The significance of induction was as follows: CON versus ACM in PPAR $\gamma$, $n=3$, two-tailed unpaired $t$ test, ${ }^{*} p=0.0310, t$ values $(t=5.548)$. CON versus HN in PPAR $\gamma, n=4$, two-tailed unpaired $t$ test, ${ }^{*} p=0.0337$, $t$ values $(t=2.741)$. CON versus ACM in Mn-SOD, $n=4$, two-tailed unpaired $t$ test, ${ }^{*} p=0.0289, t$ values $(t=$ 2.857). CON versus HN in Mn-SOD, $n=4$, two-tailed unpaired $t$ test, ${ }^{*} p=0.0209, t$ values $(t=3.109) . \boldsymbol{B}, \mathrm{Mn}-\mathrm{SOD}$ and PPAR $\gamma$ protein levels in the microglia. ACM after removing Mt (Mt depleted ACM; mdACM, by filtration with $0.22 \mu \mathrm{m}$ filter alone) or in combination with $\mathrm{HN}$ neutralizing antibody ( $\mathrm{Ab}-\mathrm{HNr}, 100 \mathrm{ng}$ ) was added to cultured microglia and incubated for $24 \mathrm{~h}$, as above. The significant changes in PPAR $\gamma ; n=3$ or 4 . One-way ANOVA/Kruskal-Wallis test: ACM versus mdACM treatment, ${ }^{*} p=0.0486$, mean rank difference (7.0) and for mdACM versus mdACM $+\mathrm{HN},{ }^{*} p=0.0011$, mean rank difference $(-12.5)$. The significant changes in Mn-SOD; $n=3-5$. One-way ANOVA/Kruskal-Wallis test: $\mathrm{ACM}$ versus $\mathrm{mdACM},{ }^{*} p=0.0173$, mean rank difference (8.4); $\mathrm{mdACM}$ versus $\mathrm{HN},{ }^{*} p=0.0210$, mean rank difference $(-9.4)$; and $\mathrm{mdACM}$ versus $\mathrm{mdACM}+\mathrm{HN},{ }^{*} p=0.0084$, mean rank difference ( -10.73$)$. C, Gene expression profile by RT-PCR (SYBR real-time analysis) in microglia treated with ACM or HN (20 $\mu \mathrm{M})$ for $24 \mathrm{~h}$. Data are mean \pm SEM; $n=3$. One-way ANOVA/Fisher's LSD test: PPAR $\gamma,{ }^{*} p=0.0131, t$ values $(t=3.484)$ at control versus $\mathrm{HN} ; \mathrm{LPL},{ }^{*} p=0.0386, t$ values $(t=2.638)$ at control versus $\mathrm{ACM},{ }^{*} p=0.0007, t$ values $(t=6.355)$ at control versus $\mathrm{HN}$; catalase, ${ }^{*} p=0.0353, t$ values $(t=2.705)$ at control versus $\mathrm{ACM},{ }^{*} p=0.0063, t$ values $(t=4.102)$ at control versus $\mathrm{HN}$; IL- $1 \beta$, ${ }^{*} p=0.0018, t$ values $(t=6.057)$ at control versus $A C M,{ }^{*} p=0.0131, t$ values $(t=3.484)$ at control versus HN. $\boldsymbol{D}, \boldsymbol{E}$, Phagocytosis assay. Rat primary microglia were treated with $\mathrm{ACM}, \mathrm{HN}(20 \mu \mathrm{M})$, or vehicle (control) for $24 \mathrm{~h}$. Rat RBCs $\left(1 \times 10^{6} \mathrm{RBCs} /\right.$ well) were added as a phagocytic target. After $2 \mathrm{~h}$, the cells were fixed and processed for immunostaining to determine the number of engulfed RBCs. D, Representative confocal image of microglia/CD11b ${ }^{+}$(green) containing engulfed RBCs (red). Scale bar, $10 \mu \mathrm{m}$. $E$, Index of phagocytosis illustrating increased levels with $A C M$ or HN treatment. Data are mean $\pm S E M ; n=3$ or 4 per group. One-way ANOVA/Bonferroni's test: ${ }^{*} p=0.0013$, CON versus ACM, $t$ values $(t=6.227) ;{ }^{*} p=0.0002$, CON versus $\mathrm{HN}, t$ values $(t=$ 8.228). CON, Control.

added to microglia and incubated for $24 \mathrm{~h}$. Western blotting revealed that PPAR $\gamma$ and Mn-SOD were significantly upregulated by $\mathrm{HN}$ or ACM (Fig. 8A). However, removal of Mt from ACM (mdACM) by filtration before ACM transfer significantly reduced these responses in microglia (Fig. $8 B$, column 2), suggesting that PPAR $\gamma$ and $\mathrm{Mn}-\mathrm{SOD}$ induction are achieved through Mt transfer rather than small particles or soluble factor(s) present in ACM. Interestingly, addition of $\mathrm{HN}$ to mdACM significantly restored Mn-SOD and PPAR $\gamma$ induction (Fig. $8 B$, columns 1,2 , and 4 ). This effect was partially ameliorated with an antibody targeting HN (Fig. 8B, columns 4 and 5).
Gene expression changes were analyzed to further clarify microglial responses to $\mathrm{HN}$ or ACM. The expression of $\operatorname{PPAR} \gamma$ and its prototypic target genes, LPL and catalase (a potent antioxidant), was increased by HN and ACM. At the tested doses, $\mathrm{HN}$ was more potent (Fig. $8 C)$. Overall, these data suggest that $\mathrm{HN}$ and $\mathrm{Mt}$ affect PPAR $\gamma$ signaling in microglia. Interestingly, HN and ACM significantly reduced IL- $1 \beta$ expression, the outcome that could occur upon increased $\operatorname{PPAR} \gamma$ signaling that normally leads to anti-inflammatory responses (Sundararajan and Landreth, 2004). There was no significant effect on IL-10 ( $p=0.8572$ in Control vs ACM, $p=0.5483$ in Control vs $\mathrm{HN})$. Considering the unaltered expression of CD68 and microglial cell counting (data not included), $\mathrm{HN}$ or Mt did not affect the number of microglia, suggesting no obvious effect on proliferation. Overall, these data suggest that $\mathrm{HN}$ and astrocyte-derived Mt are capable of inducing a "reparative" microglial phenotype.

\section{HN or Mt enhance microglial phagocytic activity toward RBCs: in vitro hematoma cleanup model}

Enhanced phagocytic function is one of the microglial responses to PPAR $\gamma$ activation (X. Zhao et al., 2007b). Together with the in vivo hematoma clearance data (Fig. $3 C$ ), the results suggest that $\mathrm{HN}$ and $\mathrm{Mt}$ could regulate the phagocytic function of microglia. To test this question, we used primary microglia treated with $\mathrm{ACM}$ or $\mathrm{HN}$ for $24 \mathrm{~h}$ before adding RBCs as a phagocytic target. After $2 \mathrm{~h}$ of incubation, the numbers of internalized RBCs per $\mathrm{CD}_{11 \mathrm{~b}^{+}}$microglia were counted under a confocal microscope. An example of microglia containing engulfed RBCs is shown in Figure 8D. HN and ACM effectively increased the numbers of engulfed RBCs by microglia compared with vehicle control (Fig. 8E). This result suggests that both $\mathrm{HN}$ and astrocytic Mt can enhance microglial phagocytic activity.

\section{Mt from HN-deficient astrocytes fail to induce a healing microglial phenotype}

As discussed above, astrocytic Mt likely modulate microglia by transporting HN to microglia. Thus, we tested whether HN deficiency in astrocytes inhibited the ability of ACM to modulate microglial expression of PPAR $\gamma$ and a prototypic target gene, LPL. Rat astrocytes were transfected with $\mathrm{HNr}$-specific siRNAs (Fig. 9A), which unlike scrambled siRNA, reduced $\mathrm{HNr}$ expression in these cells (Fig. 9B). Next, ACM from $\mathrm{HNr}$-deficient and -proficient astrocytes were collected and transferred to cultured 
microglia (Fig. 9A). In contrast to ACM from astrocytes treated with scrambled siRNA, ACM from HNr-knockdown astrocytes was significantly less effective in inducing PPAR $\gamma$ and LPL expression (Fig. 9C). ACM from $\mathrm{HNr}$ knockdown was also less effective in suppressing IL- $1 \beta$ expression by microglia (Fig. 9C).

\section{Discussion}

A limited number of studies addressed the existence of Mt transfer between cells, with most assuming that the main effect is to enhance the bioenergetic capacity of the recipient cell (Spees et al., 2006; Plotnikov et al., 2010; Islam et al., 2012; Pasquier et al., 2013; Liu et al., 2014; Wang and Gerdes, 2015; Hayakawa et al., 2016, 2018). Even fewer investigated the role of Mt transfer in the CNS (Hayakawa et al., 2016, 2018). It was recently proposed that, following brain ischemia, astrocytes increase the ATP supply by transferring functional $\mathrm{Mt}$ to neurons (Hayakawa et al., 2016). However, while the beneficial effect of Mt transfer is fairly well documented, the specific factor(s) provided by transferred Mt remain unclear. This benefit can extend beyond the energetic aspect of Mt transfer. Here, we propose that astrocytic Mt-derived HN acts as a transcellular signaling molecule that can modulate "reparative" microglial functions after being secreted by astrocytes as a peptide or while being transferred within Mt. We also propose that $\mathrm{HN}$ may help reduce damage caused by $\mathrm{ICH}$.

Our study demonstrates that astrocyte-secreted Mt are taken up by microglia, where they are detected for at least several days at the intracellular locations that do not colocalize with phagosomes, suggesting that these "adopted" Mt may not be just a target for phagocytosis. Mt transfer promoted a "reparative" microglial phenotype. Importantly, we showed that HN, a small secretory peptide normally encoded by the Mt genome, may be an essential component of biological effect following Mt transfer. Astrocytic Mt or HN stimulated the expression of microglial PPAR $\gamma$ and several PPAR $\gamma$-regulated target genes with welldefined roles in controlling antioxidative, anti-inflammatory (Yi et al., 2008; Aronowski and Zhao, 2011) and phagocytic functions (Ricote et al., 1999; X. Zhao et al., 2007b, 2015c; Roszer et al., 2011; Natrajan et al., 2015). Notably, both astrocytic Mt and HN increased the phagocytic activity of microglia toward RBCs, a principal constituent of hematomas and an object of phagocytosis-mediated cleanup after ICH (X. Zhao et al., 2007b, 2009a).

Through multiple mechanisms, including iron-catalyzed Fenton's reaction, ICH leads to excessive generation of free radicals and simultaneous loss of antioxidative defenses, which leads to irreversible mitochondrial injury and oxidative brain tissue damage in the ICH-affected hemisphere (Peeling et al., 1998; Aronowski and Hall, 2005; X. Zhao et al., 2007a, 2015a,b,c). Several experimental and human studies have documented that $\mathrm{ICH}$ affects Mt stability and causes Mt dysfunction, including excessive superoxide production at the perihematoma zone (Wu et al.,
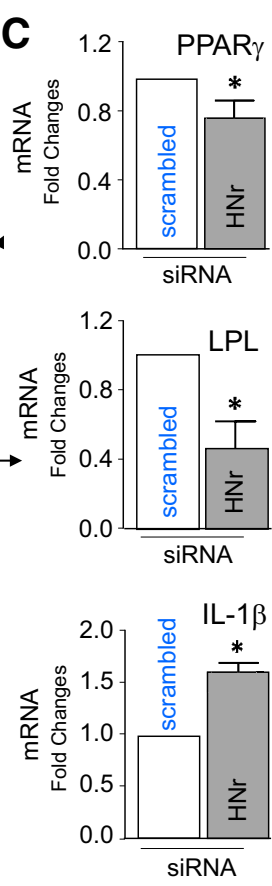

Figure 9. ACM from HN-deficient astrocytes has reduced capacity to modulate microglia. Rat astrocytes were transfected with 列 3), HNr knockdown ACM $(n=5)$, two-tailed unpaired $t$ test, PPAR $\gamma ;{ }^{*} p=0.0357, \mathrm{LPL} ;{ }^{*} p=0.0430, t$ values $(t=2.558), \mathrm{IL}-1 \beta$ ${ }^{*} p=0.0024, t$ values $(t=5.007)$

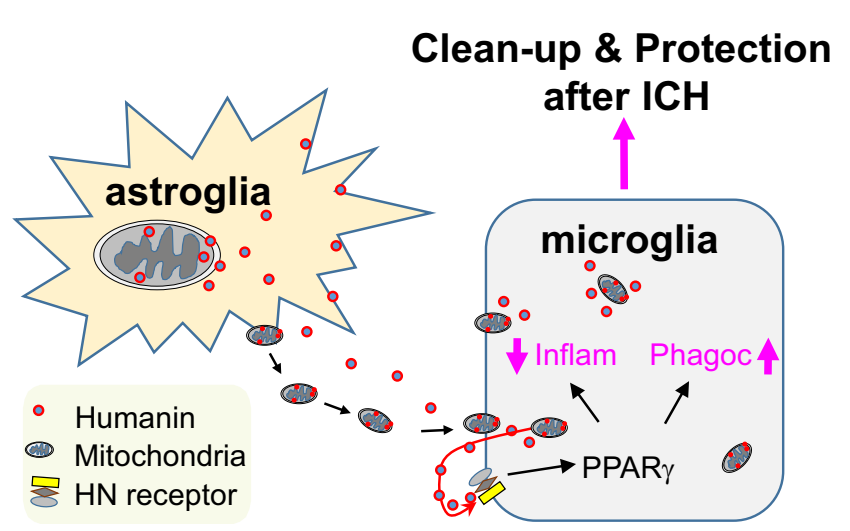

Figure 10. Astrocytes release Mt and HN that are taken up by microglia. The new microgliaadopted Mt and astrocyte-secreted HN may amplify microglial PPAR $\gamma$ production and PPAR $\gamma$ dependent gene target induction, known to promote a "healing" microglial phenotype characterized by improved phagocytic efficiency and anti-inflammatory and trophic effects.

2002; Kim-Han et al., 2006; Swanson, 2006; Lu et al., 2015; Hu et al., 2016; Qu et al., 2016). Since Mn-SOD acts as a shield preventing Mt and cells from harmful superoxides (Wu et al., 2002), any approach that upregulates Mn-SOD could mitigate ICHmediated damage. Our present data demonstrate that both astrocytic Mt and HN significantly increase the microglial expression of both Mn-SOD and the potent antioxidative enzyme catalase. Interestingly, the expression of both proteins is under transcriptional control of PPAR $\gamma$ (X. Zhao et al., 2006, 2007b, 2009c), expression of which was effectively upregulated by both HN and 
Mt. This suggests that PPAR $\gamma$ activation could play important roles in beneficial $\mathrm{HN}$ - and Mt-mediated effects after ICH. In support of this hypothesis, we further showed that microglia receiving Mt from HN knockdown astrocytes failed to upregulate the expression of PPAR $\gamma$ and its prototypic target gene LPL. This suggests that $\mathrm{HN}$ in astrocytic Mt are indeed important for the Mt transfer-induced change in microglial phenotype. In addition to acting as a carrier of presynthesized $\mathrm{HN}$, Mt entering microglia may also deliver MtDNA to allow the recipient cell to increase HN transcription. To test this model, we showed that HN mRNA and protein were significantly increased in cultured microglia following astrocytic Mt incorporation. This suggests that astrocytic Mt adopted by microglia may indeed stimulate additional HN synthesis. Although unlikely, we cannot fully exclude the possibility that Mt transfer could induce local production of $\mathrm{HN}$ mRNA from its own MtDNA.

Results from our mouse ICH model indicate that mRNA and protein levels of $\mathrm{HN}$ are robustly reduced in the $\mathrm{ICH}$-affected hemisphere. Loss extended far beyond the perihematoma tissue, suggesting that $\mathrm{ICH}$ may affect mitochondrial gene expression throughout most of the hemisphere. Presuming the previously described beneficial role of HN (Guo et al., 2003; Matsuoka, 2011; Gong et al., 2014; Cui et al., 2017; Kim et al., 2017), our results showing decreased $\mathrm{HN}$ following $\mathrm{ICH}$ suggest that $\mathrm{HN}$ supplementation could exert beneficial effects. Experiments with fluorescently tagged HN showed that systemically administered $\mathrm{HN}$ entered the brain and localized in Iba ${ }^{+}$microglia and neurons (data not included), suggesting that this cell type could be an important target for $\mathrm{HN}^{\prime}$ s biological effects. Encouraged by these results, we delivered $\mathrm{HN}$ in an $\mathrm{ICH}$ mouse model via two routes and found that it reduced neurological deficits when given intraperitoneally or intranasally. In an in vitro model of hematoma clearance, $\mathrm{HN}$ effectively increased RBC phagocytosis. This was expected based on our finding that $\mathrm{HN}$ induces expression of $\operatorname{PPAR} \gamma$, a transcription factor that we and others demonstrated to promote the phagocytic function of microglia/macrophages, including during hematoma clearance after ICH (Ricote et al., 1999; X. Zhao et al., 2007b, 2015c; Roszer et al., 2011; Natrajan et al., 2015). We believe that the present study warrants additional translational work with $\mathrm{HN}$, or synthetic $\mathrm{HN}$ analogs (Tajima et al., 2005; Xu et al., 2006; Wang et al., 2013), to determine the efficacy, appropriate doses, and therapeutic window during pleiotropic therapy for $\mathrm{ICH}$, especially assuming that $\mathrm{HN}$ could be used via a noninvasive intranasal route of delivery. Effects of age and sex also need to be examined. Collectively, these results suggest that astrocyte-derived $\mathrm{Mt}$ are transported to microglia where they stimulate the production and release of $\mathrm{HN}$ to prevent damage caused by ICH.

Since this study was designed to study astrocyte-microglia interactions, we did not focus on other types of brain cells as potential donors or recipients of $\mathrm{HN}$ and Mt. We have shown that astrocytes release $\mathrm{Mt}$; however, neurons and microglia may also release Mt that have distinct functions, be dysfunctional, or lack internalization capability. When comparing Mt from astrocytes, neurons, and microglia (ongoing studies), we observed that astrocyte-released Mt have the highest red/green fluorescence ratio by using JC-1 staining (a probe for Mt membrane potential), suggesting that these Mt are highly functional. Davis et al. (2014) showed that Mt secreted by retinal ganglion axons are internalized by the astrocytes and then degraded, suggestive of "transmitophage." Thus, neurons may also secrete Mt, but these Mt could be less healthy, have lower HN levels, and possibly be cellular waste. Preliminary experiments suggest that oxidative stress- injured neurons secrete more Mt than uninjured neurons (data not included), but these Mt also exhibited a lower JC-1 red/green ratio, suggesting that they could be dysfunctional. Platelets and mast cells also release Mt (Zhang et al., 2012; Boudreau et al., 2014). Although outside the focus of this study, we have already initiated probing for the presence and release of $\mathrm{HN}$ in cultured astrocytes, neurons, oligodendrocytes, and microglia using immune dot blot and RT-PCR. Our early results suggest that astrocytes have the highest HN expression level (data not included).

$\mathrm{HN}$ was previously shown to interact with $\mathrm{Bax}(\mathrm{Bcl} 2-$ associated X protein) by suppressing Bax translocation to $\mathrm{Mt}$, which influences mitochondrial cytochrome $\mathrm{C}$ release and triggers caspase-dependent apoptosis (Guo et al., 2003). In the context of the present study, we cannot exclude the possibility that $\mathrm{HN}$-mediated protection in ICH could also involve interference with the Bax-dependent apoptotic pathway, adding to the overall benefit through involvement of apoptotic pathways. On the other hand, it appears that apoptosis is not a key death pathway after ICH or ICH-like injury (Wang et al., 2002; Aronowski and Hall, 2005; X. Zhao et al., 2006; Zille et al., 2017), suggesting that the Bax pathway may not be the sole reason for the therapeutic benefit of $\mathrm{HN}$.

In conclusion, we demonstrated that Mt-derived $\mathrm{HN}$, whether secreted, transferred within intact Mt, or injected as synthetic HN, can promote a "reparative" microglial phenotype. Our findings suggest that $\mathrm{HN}$ and its biological properties could represent a potential therapeutic target for ICH (Fig. 10).

\section{References}

Adeoye O, Broderick JP (2010) Advances in the management of intracerebral hemorrhage. Nat Rev Neurol 6:593-601.

Aronowski J, Hall CE (2005) New horizons for primary intracerebral hemorrhage treatment: experience from preclinical studies. Neurol Res 27: $268-279$.

Aronowski J, Zhao X (2011) Molecular pathophysiology of cerebral hemorrhage: secondary brain injury. Stroke 42:1781-1786.

Boudreau LH, Duchez AC, Cloutier N, Soulet D, Martin N, Bollinger J, Paré A, Rousseau M, Naika GS, Lévesque T, Laflamme C, Marcoux G, Lambeau G, Farndale RW, Pouliot M, Hamzeh-Cognasse H, Cognasse F, Garraud O, Nigrovic PA, Guderley $\mathrm{H}$, et al. (2014) Platelets release mitochondria serving as substrate for bactericidal group IIA-secreted phospholipase A2 to promote inflammation. Blood 124:2173-2183.

Cui AL, Zhang YH, Li JZ, Song T, Liu XM, Wang H, Zhang C, Ma GL, Zhang $\mathrm{H}$, Li K (2017) Humanin rescues cultured rat cortical neurons from NMDA-induced toxicity through the alleviation of mitochondrial dysfunction. Drug Des Devel Ther 11:1243-1253.

Davis CH, Kim KY, Bushong EA, Mills EA, Boassa D, Shih T, Kinebuchi M, Phan S, Zhou Y, Bihlmeyer NA, Nguyen JV, Jin Y, Ellisman MH, MarshArmstrong N (2014) Transcellular degradation of axonal mitochondria. Proc Natl Acad Sci U S A 111:9633-9638.

Felberg RA, Grotta JC, Shirzadi AL, Strong R, Narayana P, Hill-Felberg SJ, Aronowski J (2002) Cell death in experimental intracerebral hemorrhage: the "black hole" model of hemorrhagic damage. Ann Neurol 51: 517-524.

Fuku N, Pareja-Galeano H, Zempo H, Alis R, Arai Y, Lucia A, Hirose N (2015) The mitochondrial-derived peptide MOTS-c: a player in exceptional longevity? Aging Cell 14:921-923.

Gong Z, Tas E, Muzumdar R (2014) Humanin and age-related diseases: a new link? Front Endocrinol 5:210.

Guo B, Zhai D, Cabezas E, Welsh K, Nouraini S, Satterthwait AC, Reed JC (2003) Humanin peptide suppresses apoptosis by interfering with bax activation. Nature 423:456-461.

Hashimoto Y, Niikura T, Tajima H, Yasukawa T, Sudo H, Ito Y, Kita Y, Kawasumi M, Kouyama K, Doyu M, Sobue G, Koide T, Tsuji S, Lang J, Kurokawa K, Nishimoto I (2001) A rescue factor abolishing neuronal cell death by a wide spectrum of familial Alzheimer's disease genes and Abeta. Proc Natl Acad Sci U S A 98:6336-6341.

Hashimoto Y, Kurita M, Matsuoka M (2009a) Identification of soluble 
WSX-1 not as a dominant-negative but as an alternative functional subunit of a receptor for an anti-Alzheimer's disease rescue factor humanin. Biochem Biophys Res Commun 389:95-99.

Hashimoto Y, Kurita M, Aiso S, Nishimoto I, Matsuoka M (2009b) Humanin inhibits neuronal cell death by interacting with a cytokine receptor complex or complexes involving CNTF receptor alpha/WSX-1/gp130. Mol Biol Cell 20:2864-2873.

Hayakawa K, Esposito E, Wang X, Terasaki Y, Liu Y, Xing C, Ji X, Lo EH (2016) Transfer of mitochondria from astrocytes to neurons after stroke. Nature 535:551-555.

Hayakawa K, Chan SJ, Mandeville ET, Park JH, Bruzzese M, Montaner J, Arai K, Rosell A, Lo EH (2018) Protective effects of endothelial progenitor cell-derived extracellular mitochondria in brain endothelium. Stem Cells 36:1404-1410.

Hickenbottom SL, Grotta JC, Strong R, Denner LA, Aronowski J (1999) Nuclear factor-kappaB and cell death after experimental intracerebral hemorrhage in rats. Stroke 30:2472-2477; discussion 2477-2478.

Hu X, Tao C, Gan Q, Zheng J, Li H, You C (2016) Oxidative stress in intracerebral hemorrhage: sources, mechanisms, and therapeutic targets. Oxid Med Cell Longev 2016:3215391.

Islam MN, Das SR, Emin MT, Wei M, Sun L, Westphalen K, Rowlands DJ, Quadri SK, Bhattacharya S, Bhattacharya J (2012) Mitochondrial transfer from bone-marrow-derived stromal cells to pulmonary alveoli protects against acute lung injury. Nat Med 18:759-765.

Jung JE, Kim GS, Narasimhan P, Song YS, Chan PH (2009) Regulation of Mn-superoxide dismutase activity and neuroprotection by STAT3 in mice after cerebral ischemia. J Neurosci 29:7003-7014.

Jung JE, Karatas H, Liu Y, Yalcin A, Montaner J, Lo EH, van Leyen K (2015) STAT-dependent upregulation of 12/15-lipoxygenase contributes to neuronal injury after stroke. J Cereb Blood Flow Metab 35:2043-2051.

Keep RF, Hua Y, Xi G (2012) Intracerebral haemorrhage: mechanisms of injury and therapeutic targets. Lancet Neurol 11:720-731.

Kim SJ, Xiao J, Wan J, Cohen P, Yen K (2017) Mitochondrially derived peptides as novel regulators of metabolism. J Physiol 595:6613-6621.

Kim-Han JS, Kopp SJ, Dugan LL, Diringer MN (2006) Perihematomal mitochondrial dysfunction after intracerebral hemorrhage. Stroke 37: 2457-2462.

Liu K, Ji K, Guo L, Wu W, Lu H, Shan P, Yan C (2014) Mesenchymal stem cells rescue injured endothelial cells in an in vitro ischemia-reperfusion model via tunneling nanotube like structure-mediated mitochondrial transfer. Microvasc Res 92:10-18.

Lu H, Jiang M, Lu L, Zheng G, Dong Q (2015) Ultrastructural mitochondria changes in perihematomal brain and neuroprotective effects of huperzine A after acute intracerebral hemorrhage. Neuropsychiatr Dis Treat 11: 2649-2657.

Matsuoka M (2011) Humanin signal for Alzheimer's disease. J Alzheimers Dis 24 [Suppl 2]:27-32.

Matsuoka M, Hashimoto Y (2010) Humanin and the receptors for humanin. Mol Neurobiol 41:22-28.

Muzumdar RH, Huffman DM, Atzmon G, Buettner C, Cobb LJ, Fishman S, Budagov T, Cui L, Einstein FH, Poduval A, Hwang D, Barzilai N, Cohen P (2009) Humanin: a novel central regulator of peripheral insulin action. PLoS One 4:e6334.

Nakamura T, Keep RF, Hua Y, Schallert T, Hoff JT, Xi G (2004) Deferoxamine-induced attenuation of brain edema and neurological deficits in a rat model of intracerebral hemorrhage. J Neurosurg 100:672678.

Natrajan MS, de la Fuente AG, Crawford AH, Linehan E, Nuñez V, Johnson KR, Wu T, Fitzgerald DC, Ricote M, Bielekova B, Franklin RJ (2015) Retinoid $\mathrm{X}$ receptor activation reverses age-related deficiencies in myelin debris phagocytosis and remyelination. Brain 138:3581-3597.

Paharkova V, Alvarez G, Nakamura H, Cohen P, Lee KW (2015) Rat humanin is encoded and translated in mitochondria and is localized to the mitochondrial compartment where it regulates ROS production. Mol Cell Endocrinol 413:96-100.

Pasquier J, Guerrouahen BS, Al Thawadi H, Ghiabi P, Maleki M, Abu-Kaoud N, Jacob A, Mirshahi M, Galas L, Rafii S, Le Foll F, Rafii A (2013) Preferential transfer of mitochondria from endothelial to cancer cells through tunneling nanotubes modulates chemoresistance. J Transl Med 11:94.

Peeling J, Yan HJ, Chen SG, Campbell M, Del Bigio MR (1998) Protective effects of free radical inhibitors in intracerebral hemorrhage in rat. Brain Res 795:63-70.
Plotnikov EY, Khryapenkova TG, Galkina SI, Sukhikh GT, Zorov DB (2010) Cytoplasm and organelle transfer between mesenchymal multipotent stromal cells and renal tubular cells in co-culture. Exp Cell Res 316: 2447-2455.

Qu J, Chen W, Hu R, Feng H (2016) The injury and therapy of reactive oxygen species in intracerebral hemorrhage looking at mitochondria. Oxid Med Cell Longev 2016:2592935.

Qureshi AI, Mendelow AD, Hanley DF (2009) Intracerebral haemorrhage. Lancet 373:1632-1644.

Regan RF, Panter SS (1996) Hemoglobin potentiates excitotoxic injury in cortical cell culture. J Neurotrauma 13:223-231.

Ricote M, Huang JT, Welch JS, Glass CK (1999) The peroxisome proliferator-activated receptor (PPARgamma) as a regulator of monocyte/macrophage function. J Leukoc Biol 66:733-739.

Roszer T, Menéndez-Gutierrez MP, Lefterova MI, Alameda D, Núñez V, Lazar MA, Fischer T, Ricote M (2011) Autoimmune kidney disease and impaired engulfment of apoptotic cells in mice with macrophage peroxisome proliferator-activated receptor gamma or retinoid X receptor alpha deficiency. J Immunol 186:621-631.

Spees JL, Olson SD, Whitney MJ, Prockop DJ (2006) Mitochondrial transfer between cells can rescue aerobic respiration. Proc Natl Acad Sci U S A 103:1283-1288.

Sundararajan S, Landreth GE (2004) Antiinflammatory properties of PPARgamma agonists following ischemia. Drug News Perspect 17:229236.

Swanson RA (2006) Intracerebral hematoma: beyond the mass lesion. Stroke 37:2445.

Tajima H, Kawasumi M, Chiba T, Yamada M, Yamashita K, Nawa M, Kita Y, Kouyama K, Aiso S, Matsuoka M, Niikura T, Nishimoto I (2005) A humanin derivative, S14G-HN, prevents amyloid-beta-induced memory impairment in mice. J Neurosci Res 79:714-723.

Tureyen K, Kapadia R, Bowen KK, Satriotomo I, Liang J, Feinstein DL, Vemuganti R (2007) Peroxisome proliferator-activated receptor-gamma agonists induce neuroprotection following transient focal ischemia in normotensive, normoglycemic as well as hypertensive and type-2 diabetic rodents. J Neurochem 101:41-56.

Voigt A, Jelinek HF (2016) Humanin: a mitochondrial signaling peptide as a biomarker for impaired fasting glucose-related oxidative stress. Physiol Rep 4:e12796.

Wagner KR, Sharp FR, Ardizzone TD, Lu A, Clark JF (2003) Heme and iron metabolism: role in cerebral hemorrhage. J Cereb Blood Flow Metab 23:629-652.

Wang T, Huang Y, Zhang M, Wang L, Wang Y, Zhang L, Dong W, Chang P, Wang Z, Chen X, Tao L (2013) [Gly14]-humanin offers neuroprotection through glycogen synthase kinase-3beta inhibition in a mouse model of intracerebral hemorrhage. Behav Brain Res 247:132-139.

Wang X, Gerdes HH (2015) Transfer of mitochondria via tunneling nanotubes rescues apoptotic PC12 cells. Cell Death Differ 22:1181-1191.

Wang X, Mori T, Sumii T, Lo EH (2002) Hemoglobin-induced cytotoxicity in rat cerebral cortical neurons: caspase activation and oxidative stress. Stroke 33:1882-1888.

Wu J, Hua Y, Keep RF, Schallert T, Hoff JT, Xi G (2002) Oxidative brain injury from extravasated erythrocytes after intracerebral hemorrhage. Brain Res 953:45-52.

Xi G, Fewel ME, Hua Y, Thompson BG Jr, Hoff JT, Keep RF (2004) Intracerebral hemorrhage: pathophysiology and therapy. Neurocrit Care 1:518.

Xu X, Chua CC, Gao J, Hamdy RC, Chua BH (2006) Humanin is a novel neuroprotective agent against stroke. Stroke 37:2613-2619.

Yen K, Lee C, Mehta H, Cohen P (2013) The emerging role of the mitochondrial-derived peptide humanin in stress resistance. J Mol Endocrinol 50:R11-R19.

Yi JH, Park SW, Brooks N, Lang BT, Vemuganti R (2008) PPARgamma agonist rosiglitazone is neuroprotective after traumatic brain injury via anti-inflammatory and anti-oxidative mechanisms. Brain Res 1244:164172.

Zhang B, Asadi S, Weng Z, Sismanopoulos N, Theoharides TC (2012) Stimulated human mast cells secrete mitochondrial components that have autocrine and paracrine inflammatory actions. PLoS One 7:e49767.

Zhao ST, Zhao L, Li JH (2013) Neuroprotective peptide humanin inhibits inflammatory response in astrocytes induced by lipopolysaccharide. Neurochem Res 38:581-588. 
Zhao X, Zhang Y, Strong R, Grotta JC, Aronowski J (2006) 15dprostaglandin $\mathrm{J} 2$ activates peroxisome proliferator-activated receptorgamma, promotes expression of catalase, and reduces inflammation, behavioral dysfunction, and neuronal loss after intracerebral hemorrhage in rats. J Cereb Blood Flow Metab 26:811-820.

Zhao X, Sun G, Zhang J, Strong R, Dash PK, Kan YW, Grotta JC, Aronowski J (2007a) Transcription factor Nrf2 protects the brain from damage produced by intracerebral hemorrhage. Stroke 38:3280-3286.

Zhao X, Sun G, Zhang J, Strong R, Song W, Gonzales N, Grotta JC, Aronowski J (2007b) Hematoma resolution as a target for intracerebral hemorrhage treatment: role for peroxisome proliferator-activated receptor gamma in microglia/macrophages. Ann Neurol 61:352-362.

Zhao X, Grotta J, Gonzales N, Aronowski J (2009a) Hematoma resolution as a therapeutic target: the role of microglia/macrophages. Stroke 40:S92-S94.

Zhao X, Song S, Sun G, Strong R, Zhang J, Grotta JC, Aronowski J (2009b) Neuroprotective role of haptoglobin after intracerebral hemorrhage. J Neurosci 29:15819-15827.

Zhao X, Strong R, Zhang J, Sun G, Tsien JZ, Cui Z, Grotta JC, Aronowski J (2009c) Neuronal PPARgamma deficiency increases susceptibility to brain damage after cerebral ischemia. J Neurosci 29:6186-6195.

Zhao X, Sun G, Zhang J, Ting SM, Gonzales N, Aronowski J (2015a) Dimethyl fumarate protects brain from damage produced by intracerebral hemorrhage by mechanism involving Nrf2. Stroke 46:1923-1928.

Zhao X, Sun G, Ting SM, Song S, Zhang J, Edwards NJ, Aronowski J (2015b) Cleaning up after ICH: the role of Nrf2 in modulating microglia function and hematoma clearance. J Neurochem 133:144-152.

Zhao X, Gonzales N, Aronowski J (2015c) Pleiotropic role of PPARgamma in intracerebral hemorrhage: an intricate system involving Nrf2, RXR, and NF-kappaB. CNS Neurosci Ther 21:357-366.

Zhao X, Ting SM, Liu CH, Sun G, Kruzel M, Roy-O’Reilly M, Aronowski J (2017) Neutrophil polarization by IL-27 as a therapeutic target for intracerebral hemorrhage. Nat Commun 8:602.

Zille M, Karuppagounder SS, Chen Y, Gough PJ, Bertin J, Finger J, Milner TA, Jonas EA, Ratan RR (2017) Neuronal death after hemorrhagic stroke in vitro and in vivo shares features of ferroptosis and necroptosis. Stroke 48:1033-1043. 